\title{
NOTAS SOBRE EL GÉNERO BERBERIS (BERBERIDACEAE) EN MÉXICO*
}

\author{
SERgio Zamudio
}

Instituto de Ecología, A.C., Centro Regional del Bajío, Apdo. postal 386, 61600 Pátzcuaro, Michoacán, México. sergio.zamudio@inecol.edu.mx

\section{RESUMEN}

En este trabajo se aclaran varios errores e imprecisiones históricas relacionadas con la aplicación de los nombres Berberis trifolia Schltdl. et Cham., B. ilicina Schltdl. y $B$. schiedeana Schltdl., que fueron asignados subsecuentemente a una misma especie. Se concluye que el binomio válido para esta entidad es $B$. trifolia, que fue el primero válidamente publicado aplicado a este taxon, quedando como sinónimos los dos restantes. La localidad tipo correspondiente se ubica en los límites entre los estados de Puebla y Veracruz, entre Cuauhtotolapa (La Gloria), municipio de Perote, Veracruz y Tlachichuca, municipio de Tlachichuca, Puebla. Se describe Berberis alpina Zamudio sp. nov., entidad que había estado confundida con B. schiedeana Schltdl. (sinónimo de B. trifolia). Asimismo se determina la correcta identidad de Berberis pallida Hartw. ex Benth. y de B. hartwegii Benth., de las que se proporcionan descripciones amplias; se designan lectotipos de B. hartwegii Benth., Mahonia paxii Fedde y M. zimapana Fedde.

Palabras clave: Berberidaceae, Berberis, Flora del Bajío, México.

\begin{abstract}
Some historical errors related with the application of the names Berberis trifolia Schltdl. et Cham., B. ilicina Schltdl. and B. schiedeana Schltdl., which were assigned

\footnotetext{
*Trabajo realizado con apoyo económico del Instituto de Ecología, A.C. (cuenta 20006), del Consejo Nacional de Ciencia y Tecnología y de la Comisión Nacional para el Conocimiento y Uso de la Biodiversidad.
} 
subsequently to the same entity, are clarified. Berberis trifolia is recognized as the valid name because it was the first one applied to the species. The type locality of this species is located near the border between the states of Puebla and Veracruz, between Cuauhtotolapa (La Gloria), municipality of Perote, Veracruz and Tlachichuca, municipality of Tlachichuca, Puebla. Berberis alpina Zamudio is described as a new species; it has been extensively confused with $B$. schiedeana (synonymous of $B$. trifolia); with which is not related. The correct identities of $B$. pallida Hartw. ex Benth. and B. hartwegii Benth. are determined, and lectotypes for B. hartwegii Benth., Mahonia paxii Fedde and M. zimapana Fedde are designated.

Key words: Berberidaceae, Berberis, Flora del Bajío, México.

Durante la revisión del género Berberis para la Flora del Bajío y de Regiones Adyacentes se han detectado varios problemas nomenclaturales y algunos errores e imprecisiones históricas, que han ocasionado una fuerte confusión entre los taxónomos en la asignación de los nombres correctos para algunas de las especies de este género que existen en la región; por lo que el presente artículo tiene como finalidad la corrección de dichos equívocos.

\section{IDENTIDAD DE MAHONIA TRIFOLIA, MAHONIA ILICINA Y B. SCHIEDEANA}

Un problema especialmente complicado es el que compete a la identidad de Mahonia trifolia Schltdl. et Cham., Mahonia ilicina Schltdl. y Berberis schiedeana Schltdl., nombres que se asignaron originalmente a un mismo taxon; sin embargo, con el tiempo el primero fue relegado a la sinonimia de varias especies y los dos últimos han sido incorrectamente usados por algunos autores para nombrar plantas diferentes a la que se aplicó el binomio por primera vez, como se narra a continuación.

En mayo de 1830 Schlechtendal y Chamisso (Linnaea 5: 211-212) describieron Mahonia trifolia con base en un espécimen estéril colectado en septiembre de 1828 por C. J. W. Schiede en las planicies entre Guantotalapa y Tlachichuca. La planta fue caracterizada como un "arbusto con hojas imparipinnadas, largamente pecioladas, con uno a dos pares de folíolos, los folíolos ovados, de margen sinuado, con 4 a 6 dientes espinosos, coriáceos, conspicuamente venoso-reticulados, fuertemente armados con espinas marginales". 
Más tarde, Schlechtendal (Linnaea 10: 236, 1835) consideró que el nombre de Mahonia trifolia no era el más indicado para este taxon, ya que al examinar otros ejemplares colectados por Schiede en mayo de 1829 detrás de Perote, constató que en éstos la mayoría de las hojas estaban compuestas por 5 a 7 pares de folíolos. Pensando que todo el material pertenecía a la misma especie, rechazó el binomio Mahonia trifolia y propuso sustituirlo por el de Mahonia ilicina, proporcionando la siguiente descripción: “...hojas imparipinnadas, con 1-6 pares de folíolos, el par inferior cercano a la base del pecíolo, pinnas hasta de 2 pulgadas de largo, cerca de 1 pulgada de ancho en la base, base subcuneado-ovada, suboblicua. Estípula aguda delgada membranácea unida por ambos lados a la base del pecíolo, ápice libre”.

Algunos años después el mismo autor (Schlechtendal, 1854) hizo una reevaluación de la especie y al concluir que el nombre de Mahonia ilicina tampoco era apropiado, ya que podría confundirse fácilmente con el de (Mahonia) "ilicifolia", propuso cambiarlo por el de Berberis (Mahonia) schiedeana, en reconocimiento a su descubridor. En esta ocasión el autor describió la planta como un “....arbusto glabro, de hojas imparipinnadas, con 1 a 2 pares de folíolos rígido-coriáceos, cartilaginoso-marginados, gruesamente sinuado-dentado-espinescentes, 9-11 dientes cercanos, subiguales, reticulado-venosas en ambos lados, la retícula de venas en la cara inferior evidentemente menos prominente, (el limbo) amplio casi subredondeado-ovado, base corta o muy cortamente cuneada, fija a un pecíolo común (raquis)". Schlechtendal señaló como localidad tipo "In regione frigida Mexici in los llanos inter Guantotolapa et Tlachichuca. Sept. 1828. leg. Dr. Schiede.”, y reconoció como sinónimos a Berberis pallida Benth., Mahonia ilicina Schltdl. y Mahonia trifolia Cham. \& Schldl.

El errático proceder de Schlechtendal para definir el nombre de la especie ocasionó una fuerte confusión entre los autores que posteriormente estudiaron el género Berberis, situación que persiste hasta nuestros días, pues todavía no se ha aclarado con certeza la ubicación de la localidad tipo, la identidad del taxon al que se aplicaron estos nombres y el nombre válido que le corresponde, aspectos que se abordan a continuación.

Ubicación de la localidad tipo de Mahonia trifolia, Mahonia ilicina y Berberis schiedeana

La localidad del ejemplar original de Mahonia trifolia fue referida por Schlechtendal y Chamisso como: "In planitie inter Guantotalapa et Tlachichuca" y la de Berberis schiedeana como: "In regione frigida Mexici in los llanos inter 
Guantotolapa et Tlachichuca", sin que en el protólogo se especifique el estado en que se ubica. Aunque Schlechtendal en 1835-1836 (Linnaea 10: 236), al sugerir el cambio de binomio de Mahonia trifolia por el de Mahonia ilicina menciona que revisó ejemplares colectados por Schiede detrás de Perote, esta información parece haber pasado desapercibida para otros autores, por lo que el nombre de la localidad dada por Schlechtendal y Chamisso se ha malinterpretado y distorsionando de un autor a otro a través de los años, como se muestra en el Cuadro 1.

Cuadro 1. Variación en el nombre y ubicación de la localidad tipo de Mahonia trifolia, Mahonia ilicina y Berberis schiedeana.

\begin{tabular}{|c|c|c|c|}
\hline Nombre & Localidad & Estado & Autor \\
\hline Mahonia trifolia & $\begin{array}{l}\text { In planitie inter Guantotalapa } \\
\text { et Tlachichuca }\end{array}$ & & $\begin{array}{l}\text { Schlechtendal y } \\
\text { Chamisso, } 1830\end{array}$ \\
\hline Berberis schiedeana & $\begin{array}{l}\text { In regione frigida Mexici in los } \\
\text { llanos inter Guantotolapa et } \\
\text { Tlachichuca }\end{array}$ & & $\begin{array}{l}\text { Schlechtendal, } \\
1854\end{array}$ \\
\hline Berberis ilicina & $\begin{array}{l}\text { between Guantololalpa and } \\
\text { Tlachichuca }\end{array}$ & & Hemsley, 1888 \\
\hline Berberis schiedeana & $\begin{array}{l}\text { between Guantololapa and } \\
\text { Tlachichuca }\end{array}$ & & Hemsley, 1888 \\
\hline Mahonia ilicina & $\begin{array}{l}\text { Ebene zwischen Guantololapa } \\
\text { und Tlachichuca }\end{array}$ & & Fedde, 1901 \\
\hline Mahonia schiedeana & $\begin{array}{l}\text { In den Llanos zwischen } \\
\text { Guantotalapa und Tlachichuca }\end{array}$ & & Fedde, 1901 \\
\hline Odostemon ilicinus & $\begin{array}{l}\text { From plains between } \\
\text { Guantololalpa and Tlachichilco }\end{array}$ & Veracruz & Standley, 1922 \\
\hline Odostemon trifolius & $\begin{array}{l}\text { From the plains between } \\
\text { Guantotalapa and Tlachichilco }\end{array}$ & Vereacruz? & Standley, 1922 \\
\hline Mahonia ilicina & $\begin{array}{l}\text { Between Guantolapa and } \\
\text { Tlachichuca }\end{array}$ & & Ahrendt, 1961 \\
\hline Mahonia schiedeana & $\begin{array}{l}\text { Between Guantotalapa and } \\
\text { Tlachichuca }\end{array}$ & Oaxaca & Ahrendt, 1961 \\
\hline Berberis ilicina & $\begin{array}{l}\text { Between Guantotalapa and } \\
\text { Tlachichilco }\end{array}$ & $\begin{array}{l}\text { Hidalgo - } \\
\text { Veracruz }\end{array}$ & Marroquín, 1972 \\
\hline Berberis schiedeana & $\begin{array}{l}\text { In planitie inter Guantotalapa } \\
\text { et Tlachichilco }\end{array}$ & Hidalgo & Marroquín, 1972 \\
\hline
\end{tabular}


Para esclarecer este embrollo se debe tener en cuenta que durante la estancia en México de C. J. W. Schiede de 1828 a 1836, este médico y botánico alemán exploró y colectó muestras de plantas principalmente en los estados de Veracruz y Morelos. En Veracruz tuvo como centro de sus exploraciones la ciudad de Jalapa, desde donde visitó numerosas localidades del estado incluyendo el Cofre de Perote, el volcán Pico de Orizaba, la barranca de Teocelo, la cuesta grande de Chiconquiaco y muchas otras (Stafleu y Cowan, 1985).

De acuerdo con nuestras investigaciones, la localidad tipo de Mahonia trifolia se encuentra situada cerca del pueblo Cuauhtotolapa, que Schiede registró erróneamente como Guantotalapa y al que Schlechtendal y Chamisso se refirieron como Guantotalapa o Guantotolapa y que actualmente se conoce con el nombre de La Gloria, en el municipio de Perote, Veracruz; población que se localiza muy cerca del límite entre los estados de Puebla y Veracruz. De hecho el mismo Schiede (1829) aclara esta duda en un informe sobre la región de los alrededores de Jalapa y de una excursión hacia el volcán (Pico) de Orizaba, fechado en Jalapa el 15 de noviembre de 1828, en el que menciona que al dirigirse al volcán Pico de Orizaba partieron de Jalapa y pasaron por La Joya, Las Vigas, Perote, la Hacienda de Tenestepec y Quautotolapa (sic), para llegar el tercer día de su partida a Tlachichuca, población de donde parte el camino que aún hoy es el más usado para ascender a la mencionada montaña.

De acuerdo con el protólogo de Mahonia trifolia, la planta debió haber sido colectada durante esta excursión en los terrenos planos o llanos, que se extienden entre Cuauhtotolapa (La Gloria) y Tlachichuca, en los límites entre los estados de Puebla y Veracruz. De hecho existe una colecta reciente de un Berberis de esta región (R. Acevedo y cols. 1466 (IEB, XAL)), proveniente de aproximadamente $1 \mathrm{~km}$ antes de La Gloria, en el camino entre Zalayeta y La Gloria, municipio de Perote, Veracruz, que testifica la existencia de la planta todavía en las inmediaciones de esta localidad.

Identidad de Mahonia trifolia Schltdl. et Cham.

A pesar de que Schlechtendal refirió los tres nombres a la misma especie, es oportuno aclarar que existe una notoria discrepancia entre las descripciones de $\mathrm{Ma}$ honia trifolia y Berberis schiedeana con respecto a la de Mahonia ilicina; para las dos primeras las hojas se describen como imparipinnadas, largamente pecioladas, formadas por 1 a 2 pares de folíolos; mientras que para la última se mencionan hojas compuestas por 1 a 6 pares de folíolos, con el par inferior cercano a la base del pecío- 
lo. Estas diferencias podrían deberse a que Schlechtendal mezcló en la descripción de Mahonia ilicina, las características de los ejemplares de dos especies diferentes colectadas por Schiede, pensando que ambas pertenecían a Mahonia trifolia.

Con la excepción de Fedde (1901), quién revisó el material tipo involucrado, ningún otro autor moderno había localizado los ejemplares que respaldan la aplicación de estos nombres, por lo que se pensaba que los especímenes de Schiede depositados en el Herbario de Berlín (B) habían sido destruidos durante la Segunda Guerra Mundial. Sin embargo, indagaciones recientes han revelado que el tipo de Mahonia trifolia se encuentra en B, mientras que los otros ejemplares que sirvieron de base para la descripción de Mahonia ilicina están depositados en HAL. La revisión de las fotografías de estos especímenes nos permitió confirmar que se trata de dos taxa distintos; el tipo de Mahonia trifolia depositado en B (Fig. 1A) representa una especie, mientras que los materiales colectados por Schiede detrás de Perote, etiquetados como Mahonia ilicina y depositados en HAL (Fig. 1B) pertenecen a una entidad distinta y pueden identificarse como Berberis moranensis Schult. et Schult. f.

Las colectas botánicas realizadas en años recientes en los terrenos planos o llanos que se extienden entre la Laguna de Alchichica en Puebla y Cuauhtotolapa (La Gloria) y Perote, en Veracruz, muestran que en esta región y a esta altitud sólo crece una especie de Berberis, que se caracteriza por ser un arbusto de 1 a $3 \mathrm{~m}$ de alto, con hojas compuestas imparipinnadas, con 3 a 5 folíolos coriáceos, con el margen dentado espinoso, la inflorescencia en forma de racimo, más corto que las hojas. El aspecto vegetativo de esta planta coincide completamente con la descripción que hicieron Schlechtendal y Chamisso (1830) de Mahonia trifolia y la que proporcionó posteriormente Schlechtendal (1854) de Berberis schiedeana, así como con el tipo depositado en B (Fig. 1A).

Por otra parte, queda dudosa la procedencia de los ejemplares de Schiede colectados "in regione frigida retro Perote", que se usaron en parte para la descripción de Mahonia ilicina y que han sido identificados como Berberis moranensis Schult. et Schult. f.; es posible que éstos provinieran del volcán Pico de Orizaba o de las Cumbres de Acultzingo, que son las únicas localidades en las que se he registrado esta especie en el estado de Veracruz (Marroquín, 1993).

No obstante, dado que los tres epítetos fueron publicados para nombrar a la misma especie, de acuerdo con el principio de prioridad del Código Internacional de Nomenclatura Botánica, al taxon que crece en los llanos entre Cuauhtotolapa y Tlachichuca le corresponde el nombre de Mahonia trifolia Schltdl. et Cham., que es el primero válidamente publicado aplicado a la especie; quedando como sinónimos de éste Mahonia ilicina Schltdl. y Berberis schiedeana Schltdl. Schlechtendal toda- 
Zamudio: Notas sobre el género Berberis en México

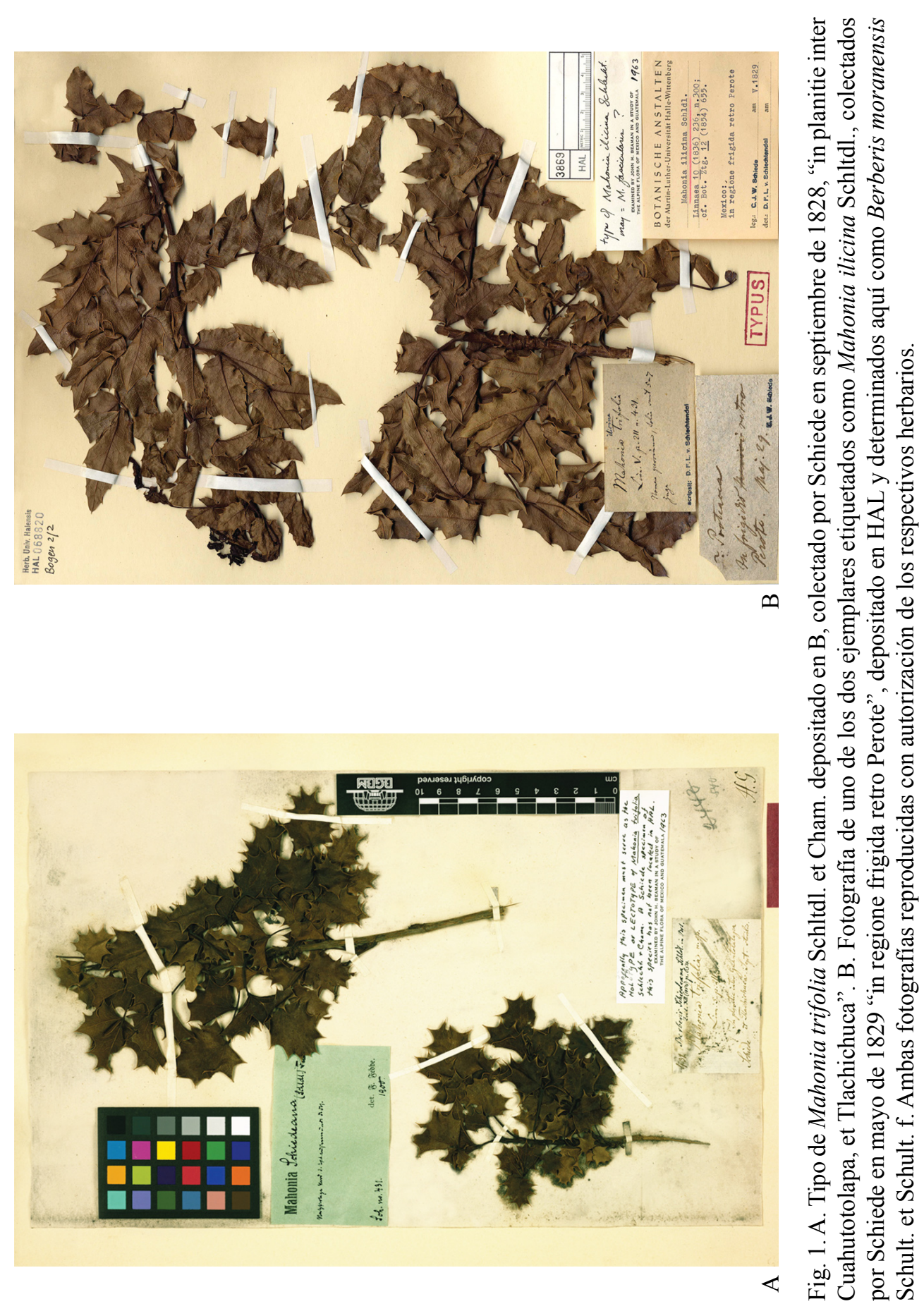


vía complicó más el asunto al considerar a Berberis pallida Hartw. ex Benth., como sinónimo de Mahonia ilicina, decisión errónea, ya que $B$. pallida es una especie distinta, como se verá más adelante.

Confusión en el uso y aplicación de los nombres

Mahonia trifolia Schltdl. et Cham. A finales de 1830, año en que fue descrita Mahonia trifolia, Schultes y Schultes f., transfirieron esta especie al género Berberis, con la combinación B. trifolia (Schltdl. et Cham.) Schult. et Schult. f. (Schultes y Schultes, 1830). Posteriormente, excepto porque Standley lo reconoció en 1922, el nombre de Mahonia trifolia, al ser rechazado por Schlechtendal pronto cayó en desuso, permaneciendo inmerso en la sinonimia de diferentes especies, principalmente de Berberis schiedeana Schltdl., Berberis ilicina (Schltdl.) Hemsl., así como también de Mahonia eutriphylla Fedde (Ahrendt, 1961). En cambio, los binomios Mahonia ilicina y Berberis schiedeana se han mantenido hasta nuestros días, aunque se adjudicaron a otras especies. A continuación se narran los cambios que han sufrido los dos últimos nombres después de la publicación original.

Mahonia ilicina Schltdl. En la Biologia Centrali-Americana, Hemsley (1888), reconoció el nombre de Mahonia ilicina Schltdl. y lo transfirió a Berberis ilicina (Schltdl.) Hemsl., señalando como sinónimo de éste a Mahonia trifolia Schltdl.

De manera similar, Fedde (1901), aceptó el binomio Mahonia ilicina Schltdl.; sin embargo, su descripción se aleja de la original de Mahonia ilicina en dos aspectos fundamentales: el primero es que señala que las hojas son compuestas por 4 (rara vez 3- o bien 5) pares de folíolos, el par inferior menor que los demás y distante 1-2.5 $\mathrm{cm}$ de la base del pecíolo. La segunda y más importante es que este autor afirma que la inflorescencia es una panícula más larga que las hojas.

Aquí es importante aclarar que Mahonia trifolia, Mahonia ilicina y Berberis schiedeana se dieron a conocer a partir de ejemplares estériles, por lo que las estructuras reproductoras no se incluyeron en su descripción. Al adjudicarle a esta especie una inflorescencia paniculada, Fedde cambió completamente su identidad, ya que como hemos mencionado con anterioridad, las plantas colectadas entre Cuauhtotolapa (La Gloria) y Tlachichuca tienen flores agrupadas en racimos.

En consecuencia, además de los ejemplares de Schiede 300 (sic 431) de los llanos entre Guantotalpa (sic) y Tlachichuca, y de Schiede s.n., detrás de Perote, Fedde adscribió a este taxon los especímenes de Ehrenberg 977, obtenidos cerca de los baños de Atotonilco el Grande, Hidalgo; de Karwinski 41, de cerca del rancho Palo 
Blanco, y de Schiede s.n., de cerca de Encarnación, todos ellos correspondientes a otra especie.

Standley (1922), en su obra "Trees and shrubs of Mexico", trata a esta especie como Odostemon ilicinus (Schltdl.) Standl., y señala como sinónimos a Mahonia ilicina Schltdl. y Berberis ilicina (Schltdl.) Hemsley. Según este autor, se trata de un arbusto de 0.5 a $3 \mathrm{~m}$ de alto; con hojas de 11 a 15 folíolos, que se distribuye en Veracruz e Hidalgo, y menciona que el tipo proviene: "from plains between Guantololapa (sic) and Tlachichilco (sic), Veracruz".

Ahrendt (1961), siguiendo a Fedde (1901), también reconoció a Mahonia ilicina Schltdl. y la describe con hojas formadas por (3-)4(-5) pares de folíolos, el par inferior 1 a $2.5 \mathrm{~cm}$ arriba de la base del raquis e inflorescencias en panículas de 10 a $15 \mathrm{~cm}$ de largo. Le asigna ejemplares de entre Guantolapa (sic) y Tlachichuca, Schiede 300; de Atotonilco, Hidalgo, Ehrenberg 977 y de cerca de La Encarnación, Hidalgo, Schiede s.n.

Por su parte, Marroquín (1972) aceptó el nombre de Berberis ilicina (Schltd1.) Hemsl., especie a la que describe como un arbusto de 1 a $3.5 \mathrm{~m}$ de alto, con hojas de 7 a $15 \mathrm{~cm}$ de largo, compuestas por 5 a 11 folíolos, el par inferior unido 0.5 a $2 \mathrm{~cm}$ de la base del pecíolo, margen 4 a 7 espinoso; inflorescencias en panículas densas de 10 a $20 \mathrm{~cm}$ de largo; bayas globosas, azul pruinosas, sin estilo.

El ejemplar tipo lo cita del estado de Hidalgo (sic), between Guantotalapa (sic) and Tlachichilco (sic), Veracruz, Schiede 300. Los demás especímenes que registra para la especie provienen de los estados de Hidalgo y San Luis Potosí.

Marroquín (1972) menciona también que Fedde (1901) había resaltado la estrecha semejanza entre Berberis ilicina y B. pallida; y en efecto, a partir de que este último autor incorpora en la descripción de Berberis ilicina una inflorescencia paniculada e incluye plantas de Hidalgo en las excicatae, se inició la práctica errónea de los autores de incluir bajo el nombre de B. ilicina (Schltdl.) Hemsl., plantas de diferentes estados que más bien pertenecen a Berberis pallida. Hartw. ex Benth.

Berberis schiedeana Schltdl. Hemsley (1888), en la Biologia Centrali-Americana incluyó a Berberis schiedeana Schltdl., mencionando como localidad: "South Mexico, los llanos, between Guantotolapa (sic) and Tlachichuca (Schiede)", sin dar una descripción ni mencionar más ejemplares.

Más tarde Fedde (1901), en su monografía del género Mahonia, también reconoció la especie Berberis schiedeana Schltdl., a la que trató como Mahonia schiedeana (Schltdl.) Fedde, asignándole como sinónimo el nombre de Mahonia trifolia 
Schltdl. et Cham. Este autor refiere para la especie además del ejemplar de Schiede colectado en 1828 en los Llanos entre Guantotalapa (sic) y Tlachichuca, especímenes provenientes de la Sierra de las Cruces (Pringle 6218) y de 40 millas al sur de Saltillo (Palmer 14); este último fue posteriormente asignado a Berberis eutriphylla (Fedde) Müller por Marroquín (1972).

La inclusión de material de lugares distintos a la localidad tipo de la especie, sobre todo del proveniente de la Sierra de las Cruces, influyó para cambiar el concepto que de esta especie tendrían los investigadores en el futuro, centrándolo en las plantas que habitan las cimas de las montañas más altas del centro de México, principalmente del Eje Volcánico Transversal.

Así, Standley (1922), en su obra "Trees and shrubs of Mexico", trata a esta planta como Odostemon trifolius (Schltdl. et Cham.) Standl., asignándole en la sinonimia a Mahonia trifolia Schltdl. et Cham., Berberis trifolia (Schltdl. et Cham.) Schult. et Schult. f., Berberis schiedeana Schltdl. y Mahonia schiedeana (Schltdl.) Fedde. Este autor indica que la especie se distribuye en los estados de Hidalgo y México y que el tipo procede: "from the plains between Guantotalapa (sic) and Tlachichilco (sic) (Veracruz?)". Standley describe a la planta como "un arbusto bajo, a veces postrado; folíolos ovados, de 2 a $3 \mathrm{~cm}$ de largo, muy espinosos; fruto azul", y comenta que asciende en la Sierra de las Cruces hasta $3600 \mathrm{~m}$ de altitud.

Es claro que Standley se refiere aquí ya francamente a los pequeños arbustos que crecen en ambientes alpinos y subalpinos en la parte alta de los volcanes del centro de México.

Por su parte, Ahrendt (1961) en su revisión de Berberis y Mahonia, reconoció a Mahonia schiedeana, y siguiendo a Fedde (1901), le asigna ejemplares de la Sierra de las Cruces, México, Pringle 6218 (K); barranca arriba de Santa Fe, Hidalgo (sic), Pringle 6975 (K); entre Guantotalapa (sic) and Tlachichucha (sic), Oaxaca (sic), Schiede, 1828 (Type) y 40 miles south of Saltillo, Nuevo León (sic Coahuila). Como se ha mencionado, el ejemplar de Palmer 14, (NY, GH), colectado en la Sierra Madre, 40 mi. S Saltillo, 22-30 Mar. 1880, más tarde fue incluido por Marroquín (1972) en las excicatae de Berberis eutriphylla (Fedde) Müller.

Marroquín $(1972,1993)$ también reconoció a Berberis schiedeana Schltdl, a la que adjudica como sinónimo el nombre de Mahonia trifolia Cham. et Schltdl. Sin embargo, la descripción que hace de la especie se ajusta más a las plantas que crecen en las altas montañas del Eje Volcánico Transversal, a las que caracteriza como un arbusto bajo con 3 a 5 folíolos por hoja, el par inferior inserto 1 a $7 \mathrm{~cm}$ de la base del pecíolo, inflorescencias en racimos fasciculados de 1 a $3 \mathrm{~cm}$ de largo, fruto ovoide, sin estilo, azul pruinoso. Menciona que el tipo proviene del estado 
de Hidalgo (sic), "In planitie inter Guantotalapa (sic) et Tlachichilco (sic)", Schiede, 1828. Los demás ejemplares que cita provienen de la Sierra de Las Cruces y de los volcanes Cofre de Perote, Iztaccíhuatl, Nevado de Toluca, Popocatépetl y Tláloc. Mientras que en su tesis doctoral (Marroquín, 1972) incluye en B. schiedeana especímenes colectados en los alrededores de la Laguna de Alchichica, en Puebla, no lo hace así en el tratamiento de la familia Berberidaceae para la flora de Veracruz (Marroquín, 1993), en donde no los menciona, a pesar de que existe una colecta de $R$. Acevedo y cols. 1466 (IEB, XAL), proveniente de aproximadamente $1 \mathrm{~km}$ antes de La Gloria, en el camino entre Zalayeta y La Gloria, municipio de Perote, Veracruz.

A continuación se proporciona una descripción más completa de Berberis trifolia basada en ejemplares depositados en los herbarios mexicanos y en observaciones de campo y se incluye un dibujo en el que se destacan las características más importantes de la especie.

Berberis trifolia (Schltdl. et Cham.) Schult. et Schult. f., in Roem. et Schult. Syst. Veg. 7(2): 1616. 1830. Fig. 2.

Mahonia trifolia Schltdl. et Cham., Linnaea 5: 211. 1830. Tipo: México, In planitie inter Guantotalapa et Tlachichuca, 1828, Schiede 431 (holotipo B, fotografía en MEXU e IEB). Fig. 1A.

Mahonia ilicina Schltdl., Linnaea 10: 236. 1835.

Berberis schiedeana Schltdl., Bot. Zeit. 12: 654. 1854.

Berberis ilicina (Schltdl.) Hemsl., Biol. Centr. Amer. Bot. 1: 23. 1879.

Mahonia schiedeana (Schltdl.) Fedde, Bot. Jahrb. Syst. 31: 90. 1901.

Odostemon trifolius (Schltdl. et Cham.) Standl., Contr. U.S. Nat. Herb. 23: 272. 1922.

Odostemon ilicinus (Schltdl.) Standl., Contr. U.S. Nat. Herb. 23: 270. 1922.

Arbusto erecto, de 1 a $3 \mathrm{~m}$ de alto; hojas compuestas imparipinnadas, de 5 a 10(14) $\mathrm{cm}$ de largo, con 3 a 5 folíolos, los laterales peciolulados, el terminal casi siempre sésil, pecíolo de 1.5 a $6 \mathrm{~cm}$ de largo, el raquis del folíolo terminal puede estar ausente o alcanzar hasta $2 \mathrm{~cm}$ de largo; limbo ovado, elíptico a ampliamente ovado, de 2 a $5 \mathrm{~cm}$ de largo por 1.8 a $4 \mathrm{~cm}$ de ancho, base cuneada, ápice agudo, mucronado-espinoso, margen dentado-espinoso, con 3 a 4(5) espinas por lado, las espinas de 2 a $3 \mathrm{~mm}$ de largo, superficie reticulado-venosa en ambas caras, la retícula de la cara inferior menos marcada, coriáceos, rígidos, verde-pardos; 


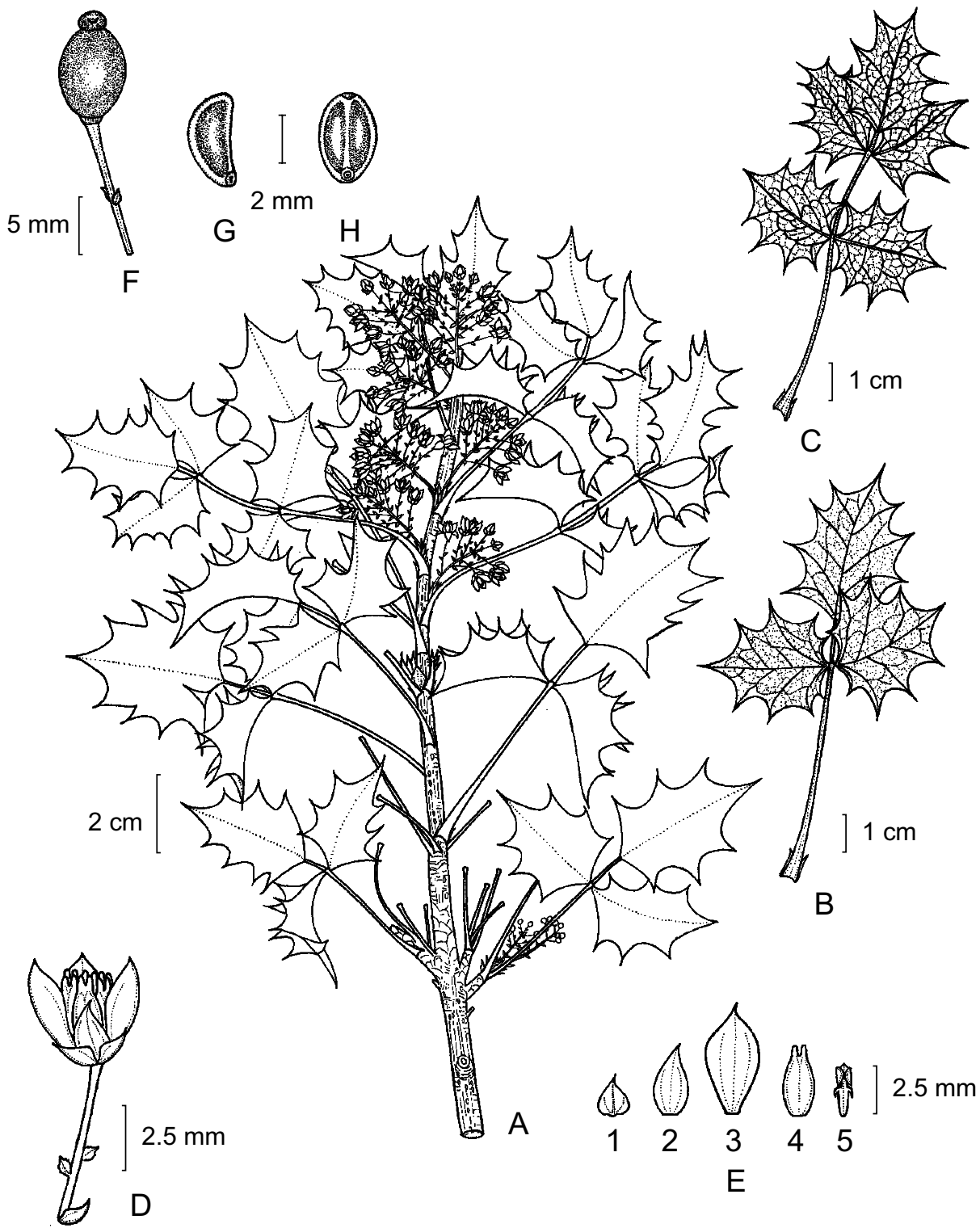

Fig. 2. Berberis trifolia (Schltdl. et Cham.) Schult. et Schult. f. A. rama con inflorescencias; B. hoja con tres folíolos; C. hoja con cinco folíolos; D. flor vista de lado; E. 1. sépalo externo, 2. sépalo medio, 3. sépalo interno, 4. pétalo, 5. estambre; F. fruto; G y H. semillas. Con base en S. Zamudio 12295 (IEB). Dibujo de Rogelio Cárdenas. 
inflorescencias en racimos axilares cortos, fasciculados, de 2 a $4 \mathrm{~cm}$ de largo, las brácteas de la base del pedúnculo de 3 a $5 \mathrm{~mm}$ de largo por 3 a $5 \mathrm{~mm}$ de ancho; pedicelos delgados, de 6 a $10 \mathrm{~mm}$ de largo, brácteas florales ovadas a suborbiculares, acuminadas, de 1.5 a $2 \mathrm{~mm}$ de largo por 1 a $1.5 \mathrm{~mm}$ de ancho, profilos ovados a lanceolados, acuminados, insertos cerca o por debajo de la mitad, excepcionalmente arriba de la mitad del pedicelo; sépalos 9, los externos ovado-lanceolados, de 1.25 a $2.5 \mathrm{~mm}$ de largo por 1 a $2 \mathrm{~mm}$ de ancho, los intermedios ovado-oblongos a ovado-lanceolados, de 2.25 a $3.5 \mathrm{~mm}$ de largo por 1.5 a $2.75 \mathrm{~mm}$ de ancho, los internos obovados, de 4 a $7 \mathrm{~mm}$ de largo por 2.5 a $4 \mathrm{~mm}$ de ancho; pétalos elípticos, emarginados en el ápice, de 4.5 a $6 \mathrm{~mm}$ de largo por 2 a $3 \mathrm{~mm}$ de ancho; estambres de 3 a $4 \mathrm{~mm}$ de largo, dentados, los dientes descendentes; fruto una baya ovoide a subglobosa, de 8 a $10 \mathrm{~mm}$ de largo, por 6 a $8 \mathrm{~mm}$ de ancho, sin estilo, de color azul pruinoso; semillas 6 o 7, obovoides a elipsoides, triquetras, de 3.5 a $4.5 \mathrm{~mm}$ de largo por 1.7 a $2.5 \mathrm{~mm}$ de ancho, café-rojizas.

Arbusto abundante, restringido a los terrenos planos o llanos, entre la Laguna de Alchichica en Puebla y Cuauhtotolapa (La Gloria) y Perote, en Veracruz. Crece en matorrales rosetófilos de Nolina, Agave, Hechtia, Juniperus deppeana y Opuntia, y en la vegetación secundaria derivada de éstos o en la orilla de los campos de cultivo. Alt. 2250-2540 m. Se ha colectado con flor de febrero a marzo y con fruto de mayo a noviembre.

Nombres comunes: chichilcuahuit, uña de gato.

Ejemplares revisados: Puebla: base del Cerro Pinto, municipio de Guadalupe Victoria, M. Cházaro B. 882 (XAL); $8 \mathrm{~km}$ al SW de Alchichica, municipio de Guadalupe Victoria, 19²1'43" N, 97²7'07" W. S. Zamudio 12295 (IEB); $12 \mathrm{~km}$ al SW de Alchichica, municipio de Guadalupe Victoria, sobre la carretera a San Salvador El Seco, J. Rzedowski 31691 (ENCB); orillas de la laguna de Alchichica, municipio de Tepeyahualco, F. Zavala Ch. 913 (ENCB, XAL); alrededores de la laguna de Alchichica, municipio de Tepeyahualco, J. I. Calzada, A. Gómez-Pompa y A. P. Vovides 2390 (ENCB); laguna de Alchichica, municipio de Tepeyahualco, 19²27' N; 97² $24^{\prime}$ W, J. I. Calzada y D. Ruiz L.13545 (XAL); laguna de Alchichica, carretera Apizaco Xalapa, municipio de Tepeyahualco, F. A. Barkley, J. Á. Villarreal y J. S. Marroquín 3808 (ENCB); alrededor de la laguna de Alchichica, municipio de Tepeyahualco, $C$. H. Ramos 50 (MEXU); laguna de Alchichica cerca de Perote, municipio de Tepeyahualco, 30.VII.1966, A. Carmona s.n. (ENCB); vaso de la laguna de Alchichica, 
carr. Zacatepec - Veracruz, municipio de Tepeyahualco, C. Muñoz B. 23 (ENCB, MEXU); Alchichica, municipio de Tepeyahualco, F. Ventura 1584 (ENCB).

Veracruz: rumbo a la laguna de Alchichica, municipio de Perote, $19^{\circ} 24^{\prime} 20^{\prime \prime} \mathrm{N}$ - 97²2'40" W, M. J. Lizama 1576 (MEXU); 8 km antes de Alchichica, municipio de Perote, W. Márquez, J. Dorantes y M. Vásquez 79 (MEXU); aproximadamente $1 \mathrm{~km}$ antes de La Gloria, camino Zalayeta - La Gloria, municipio de Perote, $R$. Acevedo R., J. L. Martínez y Pérez 1466 (IEB, XAL).

\section{DESCRIPCIÓN DE BERBERIS ALPINA}

Como consecuencia de que el binomio Berberis schiedeana Schltdl. resulta relegado a la sinonimia de $B$. trifolia Schltdl. et Cham., las plantas de Berberis que crecen en las partes altas de las montañas del centro de México (a las que hasta ahora se les había aplicado ese epíteto) se han quedado sin nombre, ya que no existe otra denominación alternativa disponible que se pueda aplicar a ellas. Por lo anterior representan una especie no descrita con anticipación, y en consecuencia es necesario darla a conocer y asignarle un nombre, lo que se hace a continuación.

Berberis alpina Zamudio, sp. nov. Fig. 3.

Mahonia schiedeana sensu Fedde, 1901, pro parte; Berberis schiedeana sensu Marroquín, 1972, non Berberis schiedeana Schltdl.

Odostemon trifolius sensu Standl., 1922, non Mahonia trifolia Schltdl. et Cham.

Tipo: México, Estado de México. Sierra de Las Cruces, C. G. Pringle 6218 (holotipo MEXU, isotipos A, MEXU, NY, UC, US).

Frutex rhizomatosus, decumbens, 0.1-1 m altus, foliis 1-2(raro-3)-jugis, longe petiolatis, petiolo (1-)3-9 $\mathrm{cm}$ longo, ad basim duabus stipulis 1-3 $\mathrm{mm}$ longis praedito, foliolis ovatis, ample ovatis vel subrotundatis, basin versus cuneatis vel rotundatis, obliquis, apicem versus acutiusculis, spinoso-acuminatis, margine undulatis, spinuloso-dentatis, dentibus utrimque 2-9(-11) triangularibus, rigide coriaceis, supra paulo nitidis caeruleo-viridibus, subtus opacis pallidis, venatio utrimque reticulata; floribus in racemis brevibus ex axillis bractearum enascentibus dispositis, (1.5-)2-3.5 mm longis; florum bracteis triangulariter acuminatis; prophyllis lanceolato-acuminatis in infima parte pedicelli; sepalis 9 in tribus seriebus dispo- 

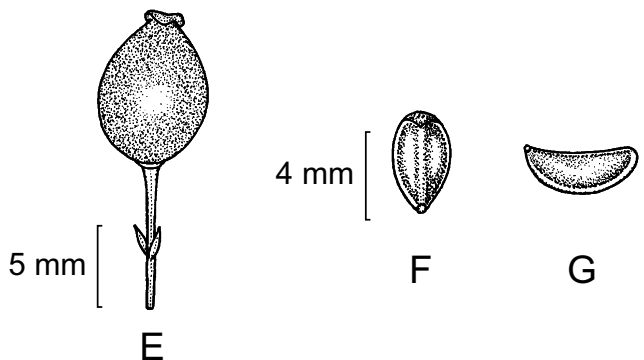

$\mathrm{E}$

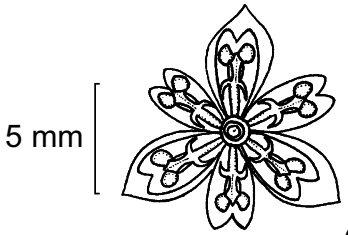

C
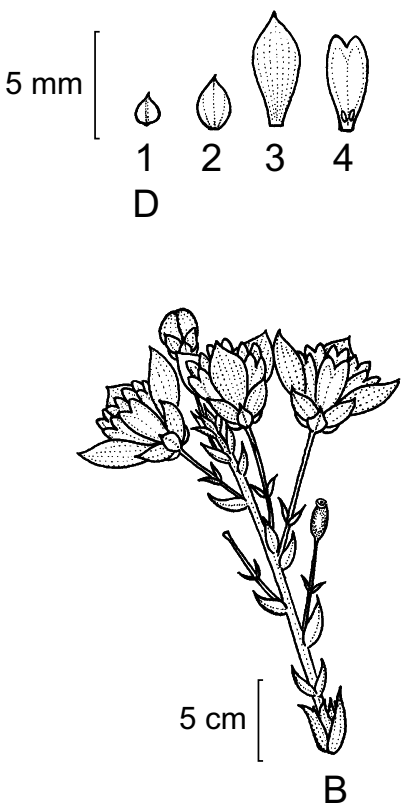

Fig. 3. Berberis alpina Zamudio. A. hábito de la planta; B. inflorescencia racemosa; C. flor vista de frente mostrando los pétalos emarginados y los estambres dentados; D. 1. sépalo externo, 2. sépalo medio, 3. sépalo interno y 4. pétalo; E. fruto; F y G. semillas. A, E, F y G, con base en S. Zamudio 7278 (IEB); B y C, con base en M. Cházaro et al. 4714 (IEB). Dibujo de Rogelio Cárdenas. 
sitis, luteis, 3 externis minimis ample ovatis (1.5-)2-3.5 mm longis, $1.5-3 \mathrm{~mm}$ latis, distincte acuminatis, 3 mediis oblongis vel obovatis, 3-6 mm longis, 2.5-4 mm latis, ad apicem rotundato-acuminatis, 3 internis obovatis, $5.5-10 \mathrm{~mm}$ longis, 3-6.5 $\mathrm{mm}$ latis, ad apicem rotundato-acuminatis; petalis 6 luteis, obovatis, $5.5-7.5 \mathrm{~mm}$ longis, 3-4 mm latis, ad apicem bilobatis; staminibus 6, (3.5-)4-6 mm longis, infra antheram dentibus duobus triangularibus praeditis; bacca ovoidea, 8-14 mm longa, 6-11 mm lata, caeruleo-pruinosa, estylosa; semina 6-7, obovoidea, triquetra, 4-5.5 mm longa, rubro-castanea.

Arbusto rizomatoso, decumbente, de 0.1 a $1 \mathrm{~m}$ de alto, a veces formando grupos densos; hojas imparipinnadas con 1 a 2 (rara vez 3) pares de folíolos sésiles, pecíolo de (1-)3 a $9 \mathrm{~cm}$ de largo, estípulas membranáceas, unidas a la base del pecíolo, el segmento apical libre subulado, el raquis del folíolo terminal puede estar ausente o alcanzar hasta $2 \mathrm{~cm}$ de largo; limbo ovado a ampliamente ovado, de (1.3-)2 a $6.5 \mathrm{~cm}$ de largo, por 1.5 a $4.3 \mathrm{~cm}$ de ancho, base cuneada, ampliamente cuneada o redondeada, oblicua, ápice agudo, mucronado-espinoso, margen dentado-espinoso, con 2 a 9(-11) espinas por lado, éstas de 1.5 a $4.5 \mathrm{~mm}$ de largo, venación fuertemente reticulada en ambas caras, coriáceo, más o menos rígido, verde-pardo, superficie brillante en el haz y más pálida y opaca en el envés; inflorescencias en racimos axilares cortos, fasciculados, de 1.5 a $4.5 \mathrm{~cm}$ de largo, las brácteas de la base del pedúnculo de 6.5 a $9 \mathrm{~mm}$ de largo por 3 a $6 \mathrm{~mm}$ de ancho; pedicelos delgados, de 4.5 a $15 \mathrm{~mm}$ de largo, brácteas florales triangulares, de 2 a 4 $\mathrm{mm}$ de largo por 1 a $2.5 \mathrm{~mm}$ de ancho, profilos lanceolados, acuminados, insertos cerca de la base del pedicelo o en la parte media; sépalos 9 dispuestos en tres series, los externos ampliamente ovados, de (1.5)2 a $3.5 \mathrm{~mm}$ de largo por 1.5 a $3 \mathrm{~mm}$ de ancho, acuminados, los intermedios oblongos u obovados, de 3 a $6 \mathrm{~mm}$ de largo por 2.5 a $4 \mathrm{~mm}$ de ancho, redondeado acuminados, los internos obovados, de 5.5 a $10 \mathrm{~mm}$ de largo por 3 a $6.5 \mathrm{~mm}$ de ancho, redondeado acuminados; pétalos obovados, emarginados en el ápice, de 5.5 a $7.5 \mathrm{~mm}$ de largo por 3 a $4 \mathrm{~mm}$ de ancho; estambres 6 , de (3.5) 4 a $6 \mathrm{~mm}$ de largo, dentados, los dientes descendentes; fruto una baya ovoide, de 8 a $14 \mathrm{~mm}$ de largo, por 6 a $11 \mathrm{~mm}$ de ancho, sin estilo, de color azul pruinoso; semillas 6 o 7, obovoides, triquetras, de 4 a $5.5 \mathrm{~mm}$ de largo, por 2 a $3 \mathrm{~mm}$ de ancho, café-rojizas.

Esta especie se distingue por ser un arbusto bajo (menor de $1 \mathrm{~m}$ de altura), postrado o decumbente, rizomatoso, por sus hojas imparipinnadas, largamente pecioladas, formadas por 3 a 5(7) folíolos (Fig. 4), por su inflorescencia en racimos 
axilares cortos de menos de $5 \mathrm{~cm}$ de largo y por habitar en ambientes alpinos y subalpinos. Crece en grupos densos sobre rocas o terrenos rocosos, en praderas alpinas y subalpinas, o en el límite altitudinal superior de los bosques de pino, pino-encino y oyamel. Alt. (2800)3000-4200 m. Se ha colectado con flor de febrero a agosto (noviembre) y con fruto de julio a noviembre.

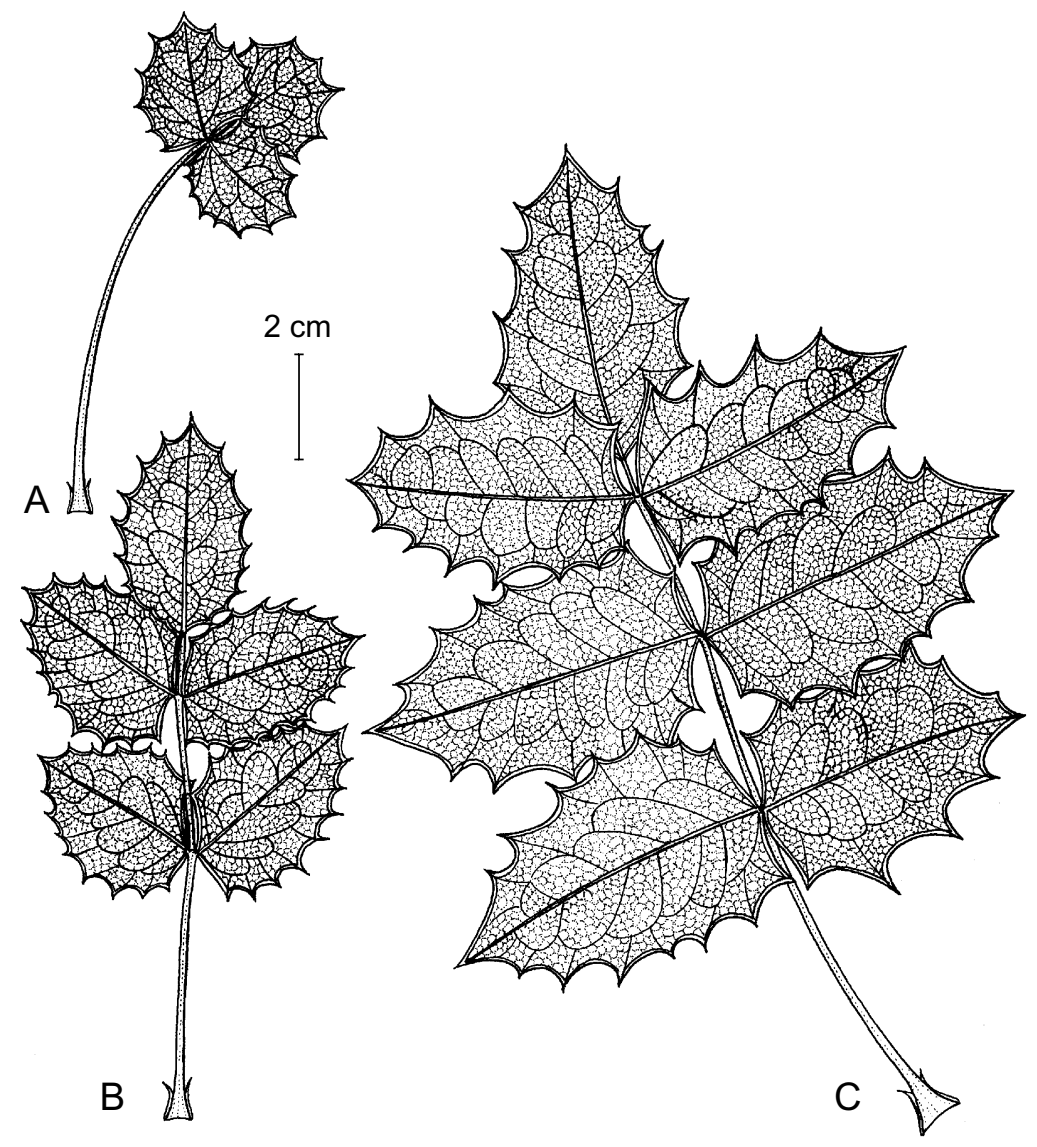

Fig. 4. Variación en las hojas de Berberis alpina Zamudio. A. hoja con tres folíolos, con base en M. Pérez G. 127 (IEB), de la Joya de Alcalican, Amecameca, México; B. hoja con cinco folíolos, con base en H. Narave y F. Vásquez 496 (XAL), del Cofre de Perote, Veracruz; C. hoja con siete folíolos, presente en muy raras ocasiones, con base en $H$. Narave y F. Vásquez 548 (XAL), del Cofre de Perote, Veracruz. Se aprecia el pecíolo largo, las estípulas unidas a la base del pecíolo y la venación por el envés. Dibujo de Rogelio Cárdenas. 
Especie endémica de las partes altas de las montañas del centro de México, principalmente del Eje Volcánico Transversal, donde es abundante; se extiende hacia el norte a los estados de Guanajuato y Querétaro, en donde es escasa y sólo se ha encontrado en el Cerro Zamorano y la Sierra del Doctor. Conocida de los estados de Gto., Qro., Hgo., Méx., D.F., Pue., Ver.

Aunque esta planta ocupa un habitat reducido en la parte superior de las altas montañas del centro de México, no parece estar amenazada ya que es resistente a la perturbación, al pastoreo y, al parecer, al fuego.

Nombres comunes: acebo, palo amarillo.

Ejemplares revisados: Distrito Federal: cima del Pico del Águila, volcán Ajusco, área natural protegida Cumbres del Ajusco, Delegación Tlalpan, J. E. Rivera H., A. Galván y A. Espinosa 3523 (MEXU); Contreras, Delegación Magdalena Contreras, Hno. E. Lyonnet 1629 (MEXU); Contreras, Delegación Magdalena Contreras, XI.1912, A. Salazar s.n. (MEXU); cañada de Contreras, arriba del $4^{\circ}$ Dínamo, Delegación Magdalena Contreras, J. L. Villaseñor 13 (MEXU); Desierto de Los Leones, Delegación Magdalena Contreras, L. Paray 590 (ENCB).

Guanajuato: Parte alta del cerro Zamorano, municipio de Tierra Blanca, $J$. Rzedowski 47797 (IEB, MEXU, XAL).

México: al $\mathrm{N}$ de Paso de Cortés, en el camino a la torre de microondas de la S.C.T., Parque Nacional Ixtaccíhuatl, municipio de Amecameca, M. A. Hernández 17 (ENCB, MEXU); en la parte E de la torre retransmisora de T.V., Las Lajas, municipio de Amecameca, A. Mancera O. 18 (ENCB); entre La Ciénega y El Salto, región de la Cabeza del Iztaccíhuatl; vertiente NW, municipio de Amecameca; J. Rzedowski 37210 (ENCB); la Joya de Alcalican, extremo SW del Ixtaccíhuatl, municipio de Amecameca, J. Rzedowski 35548 (ENCB, MEXU); camino Paso de Cortés - La Joya, entre la estación de Televisión, falda del Iztaccíhuatl, municipio de Amecameca, R. Weber 384 (ENCB); cerro Venacho, al E de Amecameca, municipio de Amecameca, J. Rzedowski 33889 (ENCB, MEXU); Telapón, municipio de Ixtapaluca, $F$. Miranda 111 (MEXU); Telapón, municipio de Ixtapaluca, $L$. $W$. Boege 59 (MEXU); cumbre del cerro Telapón, municipio de Ixtapaluca, J. D. Flores M. 297 (ENCB); Estación Experimental de Investigación y Enseñanza de Zoquiapan, $8 \mathrm{~km}$ al $\mathrm{S}$ de Río Frío, cañada Temascatitla, por camino 4, municipio de Ixtapaluca, R. Vega-Aviña 446 (IBUG, MEXU); cerro de Jocotitlán, municipio de Jocotitlán, E. Matuda 30226, 30995 (MEXU); ibid., R. Hernández M. 190 (MEXU); Zempoala, municipio de Ocuilan, E. Matuda 28145 (MEXU); alrede- 
dores de la presa Iturbide, municipio de Santiago Tlazala, J. Rzedowski 28542 (ENCB); alrededores de la presa Iturbide cerca de Santiago Tlazala, municipio de Santiago Tlazala, J. Rzedowski 30197 (ENCB); alrededores de la presa Iturbide, municipio de Santiago Tlazala, J. Rzedowski 34015 (ENCB, MEXU); Tlaloc, south side of mtn., municipio de Texcoco, J. H. Beaman 2311 (MEXU); parte alta del cerro Tláloc, municipio de Texcoco, J. Rzedowski 31553 (ENCB, IBUG); cerro Tláloc, municipio de Texcoco, E. J. Lott y T. Wendt P-73 (ENCB, MEXU); a $50 \mathrm{~m}$ de la Laguna de la Tuna, cima del Nevado de Toluca, municipio de Texcaltitlán, $X$. Madrigal y cols. S-2 (MEXU). Localidades sin municipio definido: cerro Cabeza, Hno. E. Lyonnet 3003 (MEXU); falda del volcán Ixtaccíhuatl, D. Gold 198 (ENCB, MEXU); falda SW de Ixtaccíhuatl, E. Matuda 26139 (MEXU); Ixtaccíhuatl, south side of mtn., J. H. Beaman 1959 (MEXU); Ixtaccíhuatl, 27.IV.1974, R. Banda y J. García s.n. (ENCB, MEXU).

Puebla: Face of high boulder, slopes of Ixtaccíhuatl, above Huejotzingo, E. Hernández Xolocotzi y A. J. Sharp X-169 (ENCB).

Querétaro: Sierra del Doctor, cerca de la antena de telecomunicaciones, municipio de Cadereyta, S. Zamudio y E. Pérez 9614 (IEB); cerro El Espolón, cerca del rancho El Pinalito, municipio de Cadereyta, E. Pérez y S. Zamudio 3479 (IEB); \pm 7 $\mathrm{km}$ al SE de Chavarrías, por el camino a Altamira, municipio de Cadereyta, $S$. Zamudio 7278 (IEB); parte alta del cerro Zamorano, municipio de Colón, J. Rzedowski 44435 (IEB, MEXU, QMEX, XAL); cerro El Zamorano, Puerto del Carmen, municipio de Colón, E. Pérez y G. Ocampo 4176 (IEB).

Veracruz: alrededores de la Laguna la Tilapa, Parque Nacional Cofre de Perote, municipio de Ayahualulco, H. Narave F. 912 (ENCB, MEXU, XAL); barranca El Rosario, entre El Escobillo y Los Altos, Parque Nacional Cofre de Perote, municipio de Perote, $H$. Narave F. 890 (MEXU, XAL); $1 \mathrm{~km}$ al N del Conejo, Parque Nacional Cofre de Perote, municipio de Perote, H. Narave F. y F. Vásquez B. 548 (ENCB, MEXU, XAL); $1 \mathrm{~km}$ al NW del Cofre de Perote, Parque Nacional Cofre de Perote, municipio de Perote, H. Narave F. y F. Vásquez B. 496 (MEXU, XAL); $5 \mathrm{~km}$ al NW del Cofre de Perote, Parque Nacional Cofre de Perote, municipio de Perote, H. Narave F., F. Vázquez B. y T. Mejía 742 (ENCB, IEB, XAL); cima del Cofre de Perote, municipio de Perote, G. Castillo, S. Avendaño e I. Acosta R. 18665 (IEB, MEXU); Cofre de Perote, municipio de Perote, J. Dorantes, M. Acosta, A. Calles y W. Márquez 1567 (ENCB, XAL); Cofre de Perote, municipio de Perote, A. P. Vovides e I. Meyer 356 (XAL); cercanías de la cima del Cofre de Perote, hacia la Laguna Negra, municipio de Perote, M. Cházaro B. y P. Hernández 1090 (XAL); Cofre de Perote, cerca de la cima, municipio de Perote, M. Cházaro B. y P. Hernández 4717 
(IBUG); volcán Cofre de Perote, municipio de Perote, R. Acevedo R. y B. Mostul 1483 (XAL); west side of summit of Cofre de Perote, $0.5 \mathrm{~km}$ below television towers, $12 \mathrm{~km}$ (by air) SE of town of Perote, municipio de Perote, B. F. Hansen \& M. Nee 7738 (F, US, XAL); alrededores de La Peña, Parque Nacional Cofre de Perote, municipio de Perote, H. Narave F. 804 (IEB, MEXU, XAL); cerca de La Roca, municipio de Perote, J. Dorantes 321 (MEXU, XAL).

\section{IDENTIDAD DE OTRAS ESPECIES INVOLUCRADAS EN LA CONFUSIÓN}

Es necesario aclarar también la identidad de Berberis pallida Hartw. ex Benth. y de Berberis hartwegii Benth., especies que habían estado involucradas con los nombres anteriores.

Berberis pallida Hartw. ex Benth.

Berberis pallida fue descrita en la obra Plantae Hartwegianae, publicada por Bentham en 1840, como un "arbusto de 6 a 8 pies de alto, con hojas compuestas por 11 a 13 folíolos ovados a ovado-lanceolados, ondulados, espinoso-dentados, con la base redondeada-cuneada, los inferiores poco distantes del tallo, racimos laxos de 8 a 10 pulgadas, más largos que las hojas, erectos o inclinados en el ápice. Flores blanquecinas, pétalos glandulosos en la base de la cara interna, ápice emarginado. Filamentos cortamente bidentados. Bayas globosas". El tipo señalado por Hartweg contiene la siguiente información: Cardonal, La Majada, San José del Oro, Zacualtipán et Atotonilco il Grande, Hartweg 268.

Hay que precisar que a pesar de que Hartweg definió la inflorescencia como un racimo, en las descripciones posteriores se menciona que es una panícula (Standley, 1922; Ahrendt, 1961; Marroquín, 1972), y tal carácter se pudo confirmar al revisar fotografías de los ejemplares de las colectas originales depositados en Kew (Fig. 7).

No obstante que la especie ha sido reconocida por diferentes autores (Hemsley, 1888; Fedde, 1901; Standley, 1922; Ahrendt, 1961; Marroquín, 1972), en los herbarios mexicanos se encontraron pocos ejemplares con este nombre; la mayoría de los especímenes que podrían asignarse a tal especie habían sido identificados como Berberis ilicina Schltdl., nombre que no les corresponde pues es un sinónimo de $B$. trifolia, como se ha discutido anteriormente, o se encuentran asignados a Berberis hartwegii Benth., taxon con el que se confunde por compartir varios caracteres. 
El estudio de los materiales de Berberis pallida depositados en los herbarios mexicanos ha revelado que los individuos de esta especie presentan una extraordinaria variación en el tamaño de las hojas y en el número, forma y tamaño de los folíolos. Al parecer tal versatilidad está relacionada con el ambiente en el que se desarrollan las poblaciones ya que, como puede apreciarse en la Fig. 5A, las plan-

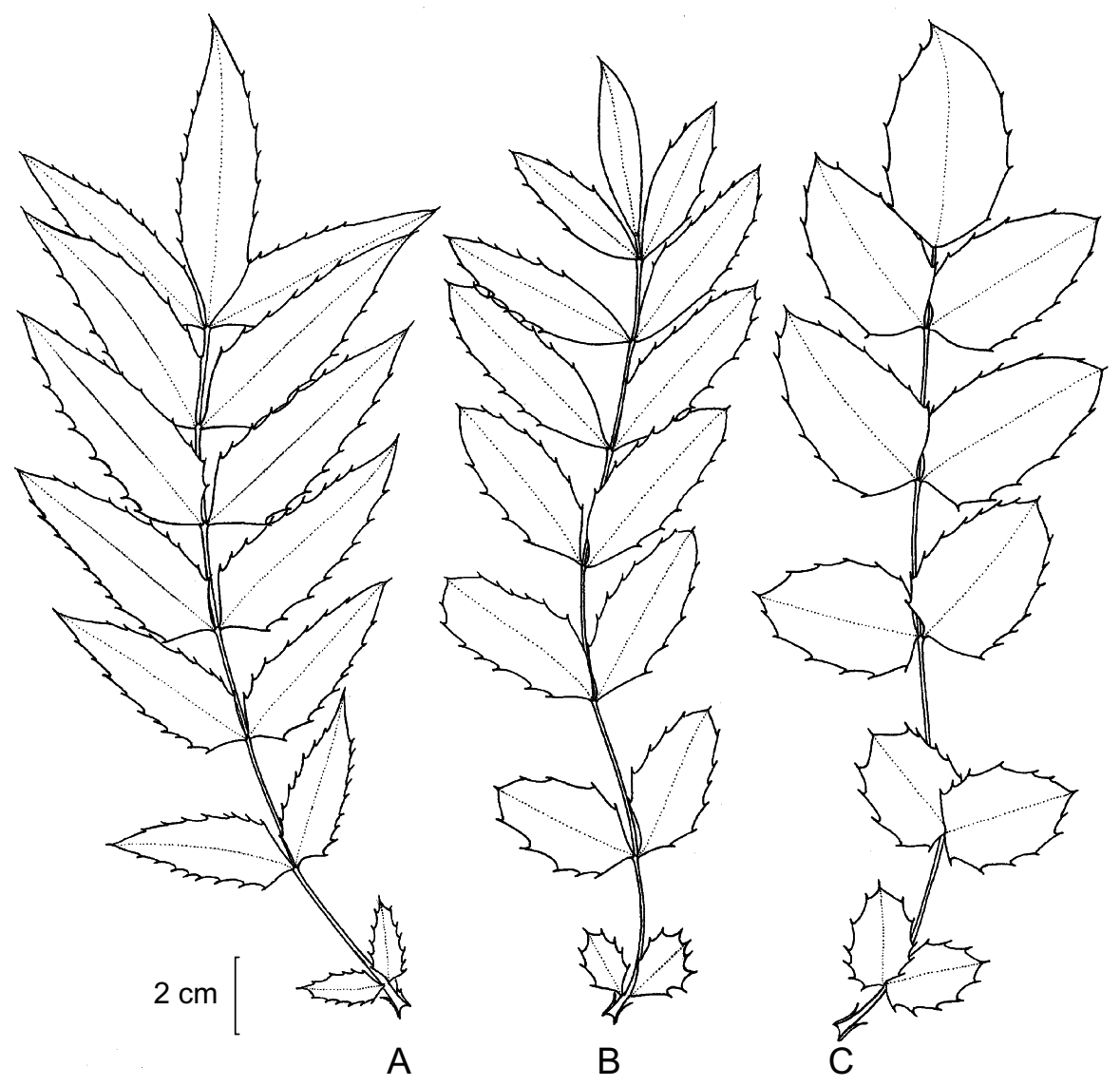

Fig. 5. Variación en las hojas de Berberis pallida Hartw. A. hoja de un ejemplar proveniente de un bosque húmedo de encinos, $3.5 \mathrm{~km}$ al $\mathrm{W}$ de La Veracruz, municipio de Cadereyta, Querétaro, S. Zamudio y E. Zamudio 10278 (IEB); B. hoja procedente de un ejemplar que crece en bosque de pino piñonero, $43.2 \mathrm{~km}$ al $\mathrm{E}$ de Xichú por el camino a Río Blanco, municipio de Xichú, Guanajuato, E. Pérez y S. Zamudio 3568 (IEB); C. hoja de un ejemplar de bosque tropical caducifolio, $\pm 2 \mathrm{~km}$ al WSW de Salvideña, municipio de Jalpan, Querétaro, E. Carranza 1859 (IEB). Dibujo de Rogelio Cárdenas. 
tas que provienen de bosques húmedos de encinos o bosques mesófilos de montaña tienen hojas más largas y delgadas, los folíolos son lanceolados, con el ápice agudo, mucronado-espinoso, los dientes y espinas son más cortos o en ocasiones están ausentes. En la Fig. 5B se muestra un ejemplar procedente de un bosque de Pinus cembroides, en el cual los folíolos son oblongo-elípticos con el ápice agudo, obtuso o redondeado, mientras que en la Fig. 5C se ilustra un espécimen que proviene de ambientes algo más áridos aún, con bosque tropical caducifolio, en el que las hojas tienen menor número de folíolos y éstos son más gruesos y coriáceos, ovados a oblongo-elípticos, con el ápice obtuso y la base redondeada. En las plantas que crecen en lugares todavía más secos, con vegetación de matorrales xerófilos, las hojas tienden a ser más cortas, con menor número de folíolos que son más anchos, oblongo elípticos, con el ápice y la base redondeada, gruesos y coriáceos y con los dientes más grandes, como puede apreciarse en la Fig. 6 y en el ejemplar tipo Fig. 7.

En la Fig. 6 se reúne una muestra más amplia de la variación encontrada en la forma y tamaño de los folíolos de $B$. pallida. Se puede apreciar que éstos pueden ser desde lanceolados con el ápice agudo-acuminado y el margen entero o dentado espinoso, hasta oblongos o suborbiculares con el ápice obtuso a redondeado, mucronado y el margen con menos dientes, los que son gruesos y llevan las espinas más largas.

Al observar por separado los extremos de esta variación se podría pensar que se trata de especies distintas; sin embargo, con el estudio de un número grande de muestras se encuentra una gradación continua entre las formas extremas, e incluso no resulta raro encontrar individuos con folíolos de diferentes formas en la misma rama (Fig. 5B).

A continuación se proporciona una descripción actualizada de esta especie, tomando en cuenta la variación observada en los ejemplares de herbario.

Berberis pallida Hartw. ex Benth., Pl. Hartw. 34. 1840. Tipo: México. Hidalgo; Cardonal, la Majada, San José del Oro, Zacualtipán, T. Hartweg 268 (Lectotipo designado por Taylor 2004, K000407229, ex Hb. Bentham). Fig. 7.

Mahonia pallida (Hartw.) Fedde, Bot. Jahrb. 31: 109. 1901.

Odostemon pallidus (Hartw.) Standl., Contr. U.S. Nat. Herb. 23: 271. 1922.

Arbusto de 1 a 3(-4) m de alto; hojas de (6-)10 a $35 \mathrm{~cm}$ de largo, con (5-)9 a 15(-17) folíolos subpeciolulados, pecíolo de 0.5 a $2(-3) \mathrm{cm}$ de largo, limbo lanceolado, ovado-elíptico a ampliamente-elíptico o ampliamente-oblongo, de 2 a 7(-11) 


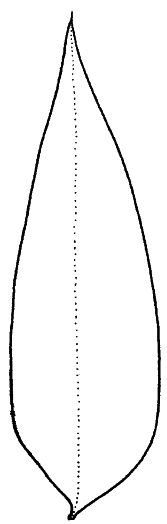

1

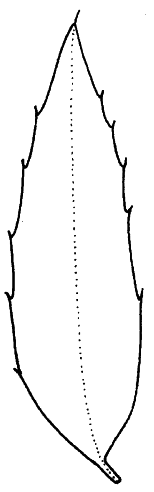

2

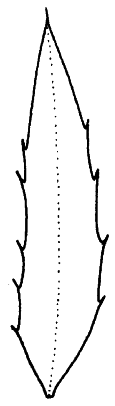

3

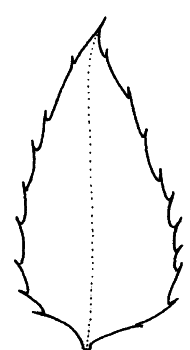

4

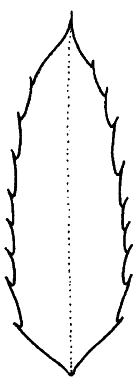

5

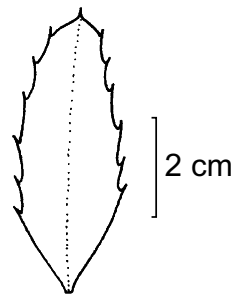

6

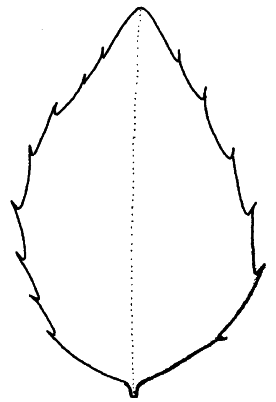

7

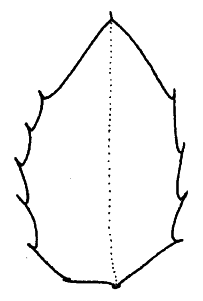

8

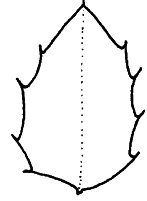

9

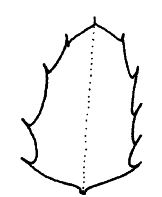

10

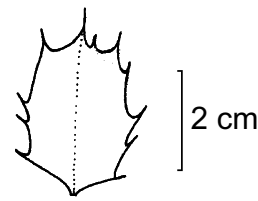

11

Fig. 6. Variación en la forma y tamaño de los folíolos de Berberis pallida Hartw. ex Benth. 1. $1.5 \mathrm{~km}$ al SW de El Naranjo, municipio de Landa, Querétaro, H. Rubio 1776 (IEB); 2. San Pedro el Viejo, municipio de Pinal de Amoles, Querétaro, E. Carranza 2190 (IEB); 3. Pinal de Amoles, Querétaro, E. Lugo 13 (IEB); $4.3 \mathrm{~km}$ al S de La Tinaja, municipio de Pinal de Amoles, Querétaro, E. Carranza 947 (IEB); 5. El Sótano de Santa María de Cocos, municipio de Arroyo Seco, Querétaro, S. Zamudio y E. Carranza 6440 (IEB); 6. Brecha Xichú-Río Blanco, municipio de Xichú, Guanajuato, E. Pérez y S. Zamudio 3568 (IEB); 7. Peñas del Bernalejo, municipio de San Luis de la Paz, Guanajuato, S. Zamudio, J. Becerra y P. Carrillo 11568 (IEB); $8.11 .2 \mathrm{~km}$ al $\mathrm{N}$ de Cuesta Colorada, municipio de Peñamiller, Querétaro, S. Zamudio y E. Zamudio 11001 (IEB). 
$\mathrm{cm}$ de largo por 1 a $5 \mathrm{~cm}$ de ancho, ápice agudo, obtuso o redondeado, mucronadoespinoso, base cuneada a redondeada, asimétrica, margen dentado-espinoso o a veces entero, con (0-)3 a 11(-14) espinas por lado, espinas de 0.5 a 3(-5) $\mathrm{mm}$ de largo, venación fuertemente reticulada, la vena media impresa en el haz y resaltada en el envés, las venas secundarias y hasta las de cuarto orden prominentes y resaltadas en el haz, formando una retícula densa que contrasta con la superficie del folíolo (Fig. 11C), café-verdosa a verde oscura, lustrosa por arriba, más pálida y opaca por debajo; inflorescencia en panículas de 10 a $30(-40) \mathrm{cm}$ de longitud, brácteas de la inflorescencia triangulares, de 4 a $15 \mathrm{~mm}$ de largo por 5 a $15 \mathrm{~mm}$ de ancho; pedicelos de 4 a $15 \mathrm{~mm}$ de longitud, brácteas florales triangulares a triangular-lanceoladas, de 2 a 10(-15) $\mathrm{mm}$ de largo por 1 a $5 \mathrm{~mm}$ de ancho; bractéolas insertas en o por debajo de la mitad del pedicelo, ovado-lanceoladas, de 1 a $3 \mathrm{~mm}$ de lago por $1 \mathrm{~mm}$ de ancho; sépalos externos ampliamente ovados a ampliamente elípticos, de 2.5 a $5 \mathrm{~mm}$ de largo por 2 a $3 \mathrm{~mm}$ de ancho, sépalos medios elípticos a ampliamente elípticos, de 5 a $6.5 \mathrm{~mm}$ de largo por 3 a $4.5 \mathrm{~mm}$ de ancho, los internos elípticos a ampliamente elípticos, de (5-) 6 a $7 \mathrm{~mm}$ de largo por 3 a $5 \mathrm{~mm}$ de ancho, pétalos obovados a elípticos, emarginados, de 4.5 a $6 \mathrm{~mm}$ de largo por 2 a $4 \mathrm{~mm}$ de ancho; estambres de 3 a $4 \mathrm{~mm}$ de largo, con dientes muy cortos debajo de la antera; fruto globoso, azul-pruinoso, de 8 a $11 \mathrm{~mm}$ de diámetro, sin estilo, semillas 2 a 9, obovoides, triquetras, de 5 a 7 $\mathrm{mm}$ de largo por 2 a $4 \mathrm{~mm}$ de ancho, de color café oscuro.

Crece en bosques de pino piñonero y enebro, encino, pino-encino y con menor frecuencia en bosque mesófilo de montaña, o en la transición de éstos con el bosque tropical caducifolio o el matorral submontano. Alt. 1000-2400 m. Se ha colectado en flor casi durante todo el año, de febrero a diciembre, sin embargo, el período de máxima floración se presenta de abril a julio, se ha colectado con fruto de junio a enero.

Especie endémica de la Sierra Madre Oriental y de otras serranías formadas por rocas calizas al sur del Eje Volcánico Transversal, en los estados de Tamps., Qro., Gto., Hgo., Pue. y Oax. Es un arbusto más o menos común; por su amplia distribución y su plasticidad adaptativa, que le permite crecer en diversos ambientes, no parece tener problemas de supervivencia.

Es muy probable que Hartweg haya colectado material de esta especie en diferentes lugares durante sus viajes en el estado de Hidalgo. En Kew existen tres ejemplares con el número Hartweg 268, pero las etiquetas manuscritas indican que fueron colectados en diferentes localidades; el pliego con número de registro K000407229 que corresponde al ejemplar designado como lectotipo por Taylor en 2004, tiene la siguiente información "Scarce, at the Cardonal, la Majada, San José del Oro, Zacual- 


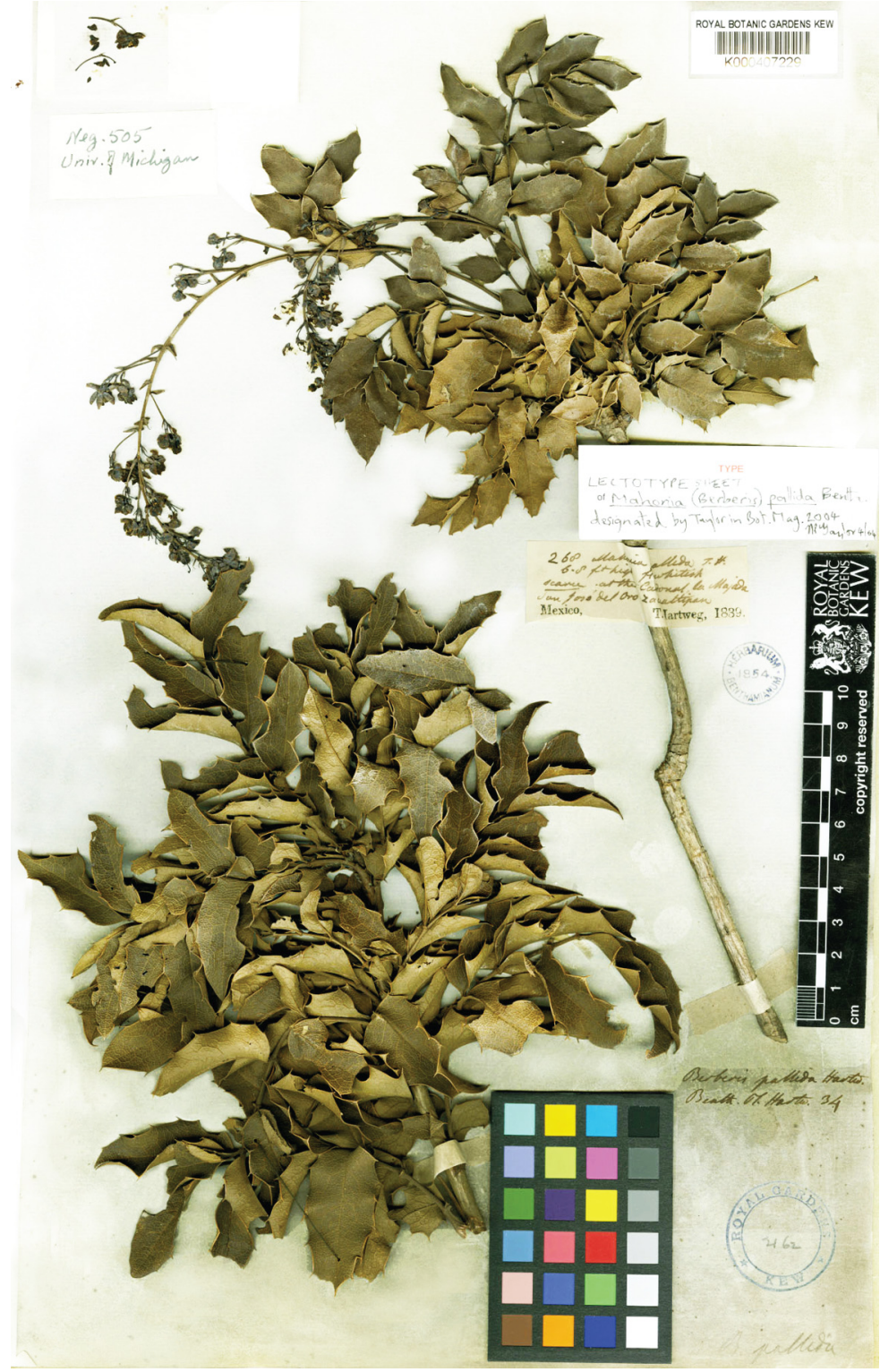

Fig. 7. Lectotipo de Berberis pallida Hartweg, T. Hartweg 268 (Kew000407229). Imagen reproducida con autorización del Herbario de los Reales Jardines Botánicos de Kew. 
tipán", cuatro lugares muy separados unos de otros. El espécimen con el número de registro K000407231, proviene de "Zacualtipan", y el que lleva el número de registro K000407232 especifica que fue colectado en la localidad de "Atotonilco il Grande."

Nombre común: palo amarillo.

Ejemplares revisados: Guanajuato: Atarjeita, municipio de Atarjea, E. Ventura y E. López 9083 (IEB); El Pinalito, por Carricillo, municipio de Atarjea, E. Ventura y E. López 9219 (IEB); Casas Viejas, $8 \mathrm{~km}$ al S de La Joya, municipio de Atarjea, E. Ventura y E. López 6510 (IEB); Peñas del Bernalejo, municipio de San Luis de la Paz, S. Zamudio, J. Becerra y P. Carrillo 11568 (IEB); El Vergel de Bernalejo, $12 \mathrm{~km}$ de Mesas de Jesús, municipio de San Luis de la Paz, E. Pérez 4786 (IEB); $43.2 \mathrm{~km}$ de Xichú por la brecha a Río Blanco, municipio de Xichú, E. Pérez y S. Zamudio 3568 (IEB).

Hidalgo: cerro de San Miguel de la Cal, $5 \mathrm{~km}$ al NW de Actopan, municipio de Actopan, L. González-Quintero 3594 (ENCB); Puente de Dios, municipio de Actopan, S. Zamudio, A. Espejo y A. R. López-Ferrari 14112 (IEB); Atotonilco el Grande, municipio de Atotonilco el Grande, 1872, M. Urbina s.n. (MEXU); Los Baños, al NE de Atotonilco el Grande, municipio de Atotonilco el Grande, $P$. Tenorio y D. Frame 11474 (MEXU); $7 \mathrm{~km}$ al $\mathrm{N}$ de Cardonal, municipio de Cardonal, L. González-Quintero 2836 (ENCB); El Mayorazgo, 4 km al NW de Cardonal, municipio de Cardonal, L. González-Quintero 2516 (ENCB); $2 \mathrm{~km}$ al W de Cardonal, municipio de Cardonal, F. González-Medrano et al. 8855 (MEXU); Tolantongo, cañada de La Piedra Cerrada, municipio de Cardonal, F. González-Medrano et al. 9464 (MEXU); barranca de Tolantongo, municipio de Cardonal, R. Hernández M. y Y. Vásquez 3778 (ENCB, MEXU); $3 \mathrm{~km}$ al N de San Cristóbal, municipio de Cardonal, F. González-Medrano et al. 9445 (MEXU); ibid. F. González-Medrano et al. 9420 (MEXU); parte alta de la barranca de Tolantongo, cerca de San Cristóbal, municipio de Cardonal, J. Rzedowski 34674 (ENCB, MEXU); barranca de Tolantongo, $42 \mathrm{~km}$ al E de Ixmiquilpan, municipio de Cardonal, F. González-Medrano et al. 9594 (MEXU); Peña del Cuervo, $5 \mathrm{~km}$ al SE de El Chico, municipio de El Chico, M. Medina C. 3099 (MEXU); tercera ampliación del ejido Julián Villagrán, municipio de Ixmiquilpan, I. Velasco R. y E. Gil A. 30 (MEXU); cañada de Arroyo Hondo, $25.9 \mathrm{~km}$ al NE de Ixmiquilpan, carretera a Tolantongo, municipio de Ixmiquilpan, $R$. Cruz-Durán et al. 4643 (MEXU); Jacala, municipio de Jacala, E. Lyonnet 1310 (MEXU); barranca Piedra Angosta, Encarnación, municipio de Zimapán, S. Zamudio, R. Ma. Murillo y A. E. Zamudio 13725, 13726, 13727, 13728, 
13730 (IEB); cañada cerca de El Salto, municipio de Zimapán, S. Zamudio, R. Ma. Murillo y A. E. Zamudio 13733 (IEB).

Oaxaca: en las montañas cercanas a Oaxaca, Distrito del Centro, C. G. Pringle 4812 (MEXU); $0.5 \mathrm{~km}$ al W del entronque carretera Oaxaca - camino a Tonaltepec, Distrito de Coixtlahuaca, $R$. Torres 5083 (MEXU); Loma Sotol al NE de Nahuizapa, municipio de Tepelmeme, distrito de Coixtlahuaca, P. Tenorio 21598 (MEXU); Mogote Ladrón, municipio de Tepelmeme de Morelos, Distrito de Coixtlahuaca, P. Tenorio 21659 (MEXU); cerro Paraje Ladrón, municipio de Villa Tepelmeme de Morelos, Distrito de Coixtlahuaca, A. Salinas T. y E. Martínez C. 7888 (MEXU); 6 $\mathrm{km}$ al $\mathrm{N}$ de Tonaltepec, municipio de Nacaltepec, Distrito de Cuicatlán, $R$. Torres y P. Tenorio 12965 (MEXU); $39 \mathrm{~km}$ al S de Cuicatlán, municipio de Nacaltepec, Distrito de Cuicatlán, R. Torres y M. L. Torres 6922 (MEXU); $50 \mathrm{~km} \mathrm{~N}$ of Oaxaca valley at Telixtlahuaca, along road to Tehuacan, Distrito de Etla, D. E. Breedlove 49611 (MEXU), cerro La Cruz o La Calera, a $11 \mathrm{~km}$ de San Marcos Arteaga, carretera a Tonalá, Distrito de Huajuapan, J. I. Calzada, E. Ortiz y L. Cervantes 18398 (MEXU); 4 km al N de Santos Reyes Tepejillo, senda hacia Xinicahua y Río Boquerón, municipio de Santos Reyes Tepejillo, Distrito de Justlahuaca, J. I. Calzada 22015 (MEXU); 4 km al N de Santos Reyes Tepejillo, municipio de Santos Reyes Tepejillo, Distrito de Justlahuaca, J. I. Calzada 21041 (MEXU); 8 km al N de Santos Reyes Tepejillo, entrada por La Cruz - Río Boquerón, municipio de Santos Reyes Tepejillo, Distrito de Justlahuaca, J. I. Calzada 19639 (MEXU); 9 miles south of Miahuatlán along road to Puerto Ángel, Distrito de Miahuatlán, D. E. Breedlove 15848 (ENCB, MICH); 7 km al NW de El Parián, camino a Huauclilla, municipio de Huauclilla, Distrito de Nochixtlán, A. Gracia-Mendoza y E. Solano 7309 (MEXU); cerro San Blas, municipio de Huauclilla, Distrito de Nochixtlán, C. Conzatti 4269 (MEXU); $26 \mathrm{~km}$ al W de Tecomavaca, brecha a Santa María Ixcatlán, Distrito de Teotitlán, P. Tenorio 18127 (MEXU); $5 \mathrm{~km}$ al S de Tecomavaca y $23 \mathrm{~km}$ al W, rumbo a Santa María Ixcatlán, Distrito de Teotitlán, A. Salinas $T$. y E. Martínez C. 6116 (MEXU); $1.5 \mathrm{~km}$ al SW de Tonaltepec, municipio de Santo Domingo Tonaltepec, Distrito de Teposcolula, F. González-Medrano et al. 1754 (MEXU);

Puebla: Chila - Zapotitlán, municipio de Chila, F. Miranda 2844 (MEXU); near Chila, municipio de Chila, 6.II.1942, G. B. Sanders s.n. (ENCB); along Hwy. 190 between Oaxaca and Izucar de Matamoros, 193 miles NW of Oaxaca, at km 194, ca. 2 miles SE of Chila, near Puebla - Oaxaca border, municipio de Chila, T. B. Croat \& D. P. Hannon 65690 (MEXU); Tlacuilosto, al S de Atzingo, municipio de Zacatlán, P. Tenorio et al. 8439 (MEXU). 
Querétaro: 3-4 km al WSW de La Florida, municipio de Arroyo Seco, E. Carranza 3085 (IEB); $2 \mathrm{~km}$ al S de La Florida, por el camino a Xichú, municipio de Arroyo Seco, E. Pérez y S. Zamudio 3264 (IEB); $\pm 10 \mathrm{~km}$ del Puerto de Ayutla, camino a Santa María de Cocos, municipio de Arroyo Seco, E. Carranza 1896 (IEB); el Sótano de Santa María de Cocos, municipio de Arroyo Seco, S. Zamudio y E. Carranza 6440 (IEB); el Sótano, $\pm 4 \mathrm{~km}$ al S de Santa María de Cocos, municipio de Arroyo Seco, A. Herrera 131 (IEB); Sótano del Barro, al S de Santa María de Cocos, municipio de Arroyo Seco, E. Carranza 2949 (IEB); alrededores de la boca del Sótano El Barro, Santa María de Cocos, municipio de Arroyo Seco, J. Treviño y J. M. Olvera 179, 371 (QMEX); 2 km al W de El Jardín, municipio de Arroyo Seco, E. Carranza 1843 (IEB); $3.5 \mathrm{~km}$ al W de La Veracruz, por el camino a La Mora, municipio de Cadereyta, S. Zamudio y E. Zamudio 10278 (IEB); \pm 3 $\mathrm{km}$ al WSW de El Lindero, municipio de Jalpan, E. Carranza 1107 (IEB); $2 \mathrm{~km}$ al WSW de Saldiveña, municipio de Jalpan, E. Carranza 1859 (IEB); $3 \mathrm{~km}$ al SE de El Lobo, municipio de Landa, L. M. Chávez 144 (IEB); 10 km al NW de El Madroño, municipio de Landa, J. Rzedowski 44106 (IEB); 8-9 km por la brecha a Tres Lagunas, municipio de Landa, E. Carranza 1708 (IEB); cerca de Tres Lagunas, municipio de Landa, J. Rzedowski 46694 (IEB); 1.5 km al SW de El Naranjo, municipio de Landa, H. Rubio 1508, 1776 (IEB); El Encinote, $1 \mathrm{~km}$ al N de Puerto del Sabino, municipio de Landa, H. Rubio 148 (IEB); El Banco, $1.5 \mathrm{~km}$ al Poniente del Puerto Hondo, municipio de Landa, P. Tenorio e H. Rubio 19204 (MEXU); Puerto Colorado, aproximadamente $6 \mathrm{~km}$ al $\mathrm{N}$ de Acatitlán de Zaragoza, municipio de Landa, S. Zamudio y E. Carranza 7170 (IEB); about $80 \mathrm{~km}$ NE of Querétaro, above Pilón on road to Pinal de Amoles, municipio de Peñamiller, R. McVaugh 10354 (MEXU, MICH, US); 1-2 km al NE de Cuesta Colorada, municipio de Peñamiller, E. Carranza 2618 (IEB); $2 \mathrm{~km}$ al $\mathrm{N}$ de Cuesta Colorada por la carretera a Pinal de Amoles, municipio de Peñamiller, S. Zamudio y E. Zamudio 11001 (IEB); Cuesta Colorada - El Madroño, municipio de Peñamiller, R. Hernández M., J. Orozco y C. Orozco 11429 (QMEX); al W de Cuatro Palos, municipio de Pinal de Amoles, E. Carranza 3158 (IEB); $\pm 1 \mathrm{~km}$ al NE de Pinal de Amoles, municipio de Pinal de Amoles, E. Carranza 520 (IEB); $2 \mathrm{~km}$ al NE de Pinal de Amoles, sobre la carretera a Jalpan, municipio de Pinal de Amoles, J. Rzedowski 43120 (IEB); ibid. E. Pérez 4348 (IEB); 3-4 km al ENE de Pinal de Amoles, municipio de Pinal de Amoles, E. Lugo 13 (IEB); Joya de Los Granadillos, $3 \mathrm{~km}$ al $\mathrm{S}$ de Los Pinos, municipio de Pinal de Amoles, S. Zamudio 6581 (IEB); al S de Los Pinos, municipio de Pinal de Amoles, E. Carranza 2480 (IEB); $3 \mathrm{~km}$ al S de La Tinaja, municipio de Pinal de Amoles, E. Carranza 947 (IEB); al W de Cuatro Palos, municipio de Pinal de 
Amoles, E. Carranza 3158 (IEB); 2-3 km al NNW de San Pedro El Viejo, municipio de Pinal de Amoles, E. Carranza 2190 (IEB); 4 km al NE de San Pedro El Viejo, sobre el camino a La Yerbabuena, municipio de Pinal de Amoles, J. Rzedowski 43034 (IEB); $1.5 \mathrm{~km}$ al SE de San Pedro Escanela, municipio de Pinal de Amoles, R. Fernández 2483 (ENCB, IEB); barrancas en los alrededores del Madroño, municipio de Pinal de Amoles, R. Hernández M., J. Orozco y C. Orozco 10183 (IEB, QMEX); ruinas Las Ranas, $2 \mathrm{~km}$ al N de San Joaquín, municipio de San Joaquín, R. Fernández 3268 (IEB); $13 \mathrm{~km}$ al NW de San Joaquín por el camino a Bucareli, municipio de San Joaquín, S. Zamudio 7299 (IEB).

Tamaulipas: $3 \mathrm{~km}$ al SW de Joya de Herrera, municipio de Bustamante, $L$. Hernández 1879 (MEXU); perfil a través de la Sierra Madre Oriental en la región de Gómez Farías, municipio de Gómez Farías, P. S. Martin y B. E. Harrell 64 (ENCB); Sierra de Guatemala, municipio de Gómez Farías, J. R. Sullivan 542 (ENCB); 2 $\mathrm{km}$ al SE de Magdaleno Aguilar, municipio de Jaumave, F. González-Medrano, A. Castellanos y P. Zavaleta 9892 (MEXU); Charco Colorado, $17 \mathrm{~km}$ al NE de rancho El Julilo, municipio de Jaumave, F. González-Medrano et al. 10445 (MEXU); 18 $\mathrm{km}$ al SW de Miquihuana, municipio de Miquihuana, F. González-Medrano 4742 (MEXU); ejido La Presita, carretera Tula-Victoria, municipio de Tula, M. Yánez 330 (MEXU); ejido Ricardo García o La Presita, km 66 carretera Tula-Victoria, municipio de Tula, M. Martínez 1179 (MEXU).

El concepto aquí aceptado de Berberis pallida, no concuerda con el de Marroquín (1972), ya que los ejemplares que este autor consideró dentro de tal especie, más bien forman parte de la variación de Berberis hartwegii Benth. En cambio, las plantas incluidas aquí en Berberis pallida coinciden con las tratadas por Marroquín (op. cit.) como Berberis ilicina Schltdl., nombre que como ya hemos discutido es un sinónimo de Berberis trifolia (Schltdl. et Cham.) Schult. et Schult. f.

El ejemplar de Berberis pallida de R. McVaugh 10354 (MEXU, MICH, US), colectado $80 \mathrm{~km}$ al NE de Querétaro, arriba del Pilón en el camino a Pinal de Amoles, fue incluido erróneamente por Marroquín (1972) como parte de Berberis zimapana (Fedde) Marroquín \& Laferr., que es un sinónimo de B. hartwegii Benth.

También es necesario comentar que en los especímenes procedentes de Puebla y Oaxaca el patrón de venación es un poco distinto del de las poblaciones de más al norte, pero esta diferencia no parece suficiente como para separarlos en otra especie; sería necesario realizar estudios más detallados y quizás con marcadores moleculares para esclarecer totalmente las relaciones entre las diversas poblaciones de Berberis pallida. 


\section{Berberis hartwegii Benth.}

Un fenómeno parecido al comentado con la especie anterior ocurre con Berberis hartwegii, taxon también dado a conocer por Bentham en 1840 en la obra Plantae Hartwegianae a partir de ejemplares colectados por Hartweg. La planta fue descrita como un "arbusto con hojas adultas de hasta $45 \mathrm{~cm}$ de largo, compuestas por 11-15 folíolos ovado-lanceolados, los inferiores cercanos a la base del pecíolo, ápice espinoso-acuminado, planos o un poco ondulados, margen espinuloso-serrulado o entero, base redondeada-truncada, superficie brillante; racimos alargados, laxos, paniculado-ramosos, con frecuencia de $30 \mathrm{~cm}$ de largo, ramas dicotómicamente alternas con 3 a 15 flores, filamentos sin dientes. El ejemplar original se cita de: Ad Contadero, inter Tulo (sic Tula) et Santa Barbara.

Antes de continuar, es necesario aclarar que la localidad tipo de esta especie se sitúa en el estado de Tamaulipas, entre Tula y Santa Bárbara y no en el estado de Hidalgo, como lo han señalado algunos autores (Standley, 1922; Ahrendt, 1961; Marroquín, 1972). La confusión se originó debido a que Bentham no indicó en el protólogo el estado de donde proceden los ejemplares que ha visto y Standley (1922) interpretó que esta localidad se encuentra situada en el estado de Hidalgo, en donde existe una importante ciudad llamada Tula. Sin embargo, al revisar el itinerario de Hartweg se comprueba que en febrero de 1838 este colector viajó de San Luis Potosí hacia Tula, Tamaulipas. McVaugh (1970: 26-27), reseña este viaje de la siguiente manera: “...el 26 de febrero él (Hartweg) dejó San Luis Potosí, el camino lo condujo casi $100 \mathrm{~km}$ al este, "por una inmensa planicie, que no ofrecía variedad (de plantas) en esa estación". Considerablemente más lejos, hacia el noreste, cerca de Tula, Tamaulipas, donde la Altiplanicie Central de México empieza a descender hacia la costa del Atlántico, encontró el campo "más próspero" y planeó permanecer allí algunas semanas, pero pronto continúo un poco más lejos hacia la vertiente del Atlántico, al rancho Los Gallitos, (...) Finalmente en marzo llegó al final de esta excursión a Santa Bárbara (ahora Ocampo, Tamaulipas)".

La revisión del material de este taxon, depositado en los herbarios mexicanos mostró una amplia variación en la forma y tamaño de las hojas y los folíolos, así como en el tamaño y número de espinas por lado, siguiendo una tendencia parecida a la descrita para Berberis pallida. En las Figs. 8 A y B se aprecia que las plantas que prosperan en ambientes húmedos con vegetación de bosque mesófilo de montaña 0 bosque húmedo de encinos son más grandes y presentan los folíolos lanceolados con el margen entero o con dientes cortos y espinas chicas, mientras que en las que crecen en ambientes más secos con vegetación de bosque de encino o de pino piñonero 
los folíolos son elípticos u oblongos, con el margen dentado (Fig. 8C). Un mayor grado de variación de los folíolos de Berberis hartwegii puede apreciarse en la Fig. 9, en donde se muestra que éstos van desde lanceolados u ovados a oblongos, elípticos o suborbiculares y que el ápice puede variar de agudo a obtuso o redondeado. Las

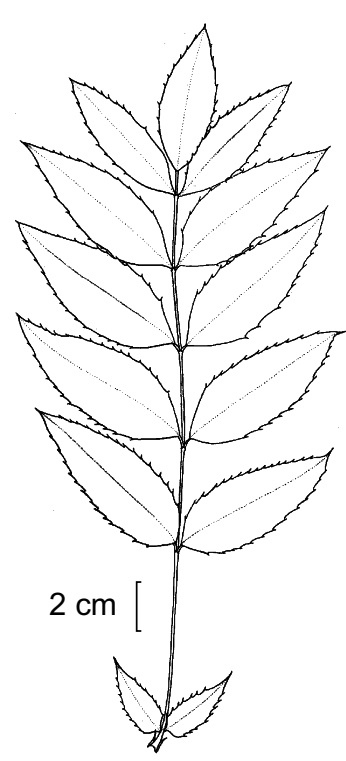

A

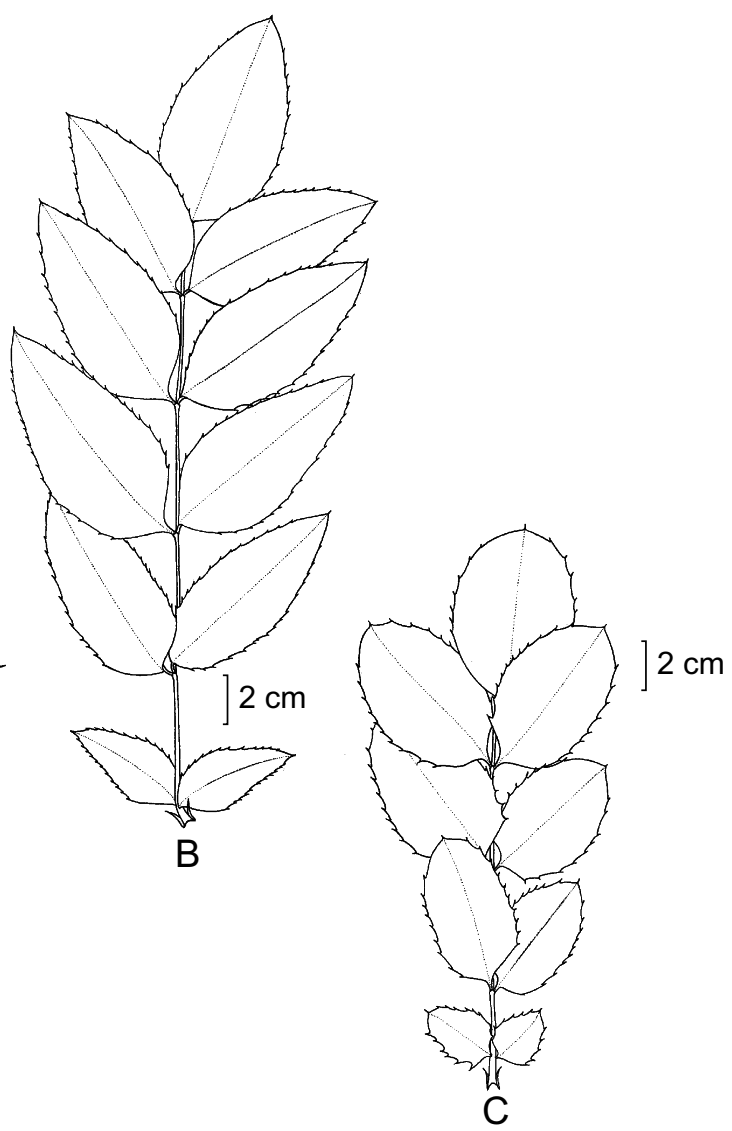

Fig. 8. Variación en las hojas de Berberis hartwegii Benth. A. hoja proveniente de bosque mesófilo de montaña, 2-3 km al N de Valle Verde, municipio de Jalpan, Querétaro, S. Servín 93 (IEB); B. hoja proveniente de bosque de encino húmedo, $\pm 3 \mathrm{~km}$ al ENE de Valle Verde, municipio de Jalpan, Querétaro, E. Carranza y A. Blanco 6061 (IEB); C. hoja proveniente de bosque de encino 4-5 km al NW de Tilaco, municipio de Landa, Querétaro, E. Carranza 1597 (IEB). Dibujo de Rogelio Cárdenas. 
plantas colectadas en el matorral submontano mantienen tallas menores y presentan folíolos que varían de ovados a oblongos o suborbiculares, con el ápice redondeado, el margen con menor número de dientes y espinas más grandes. En los ejemplares

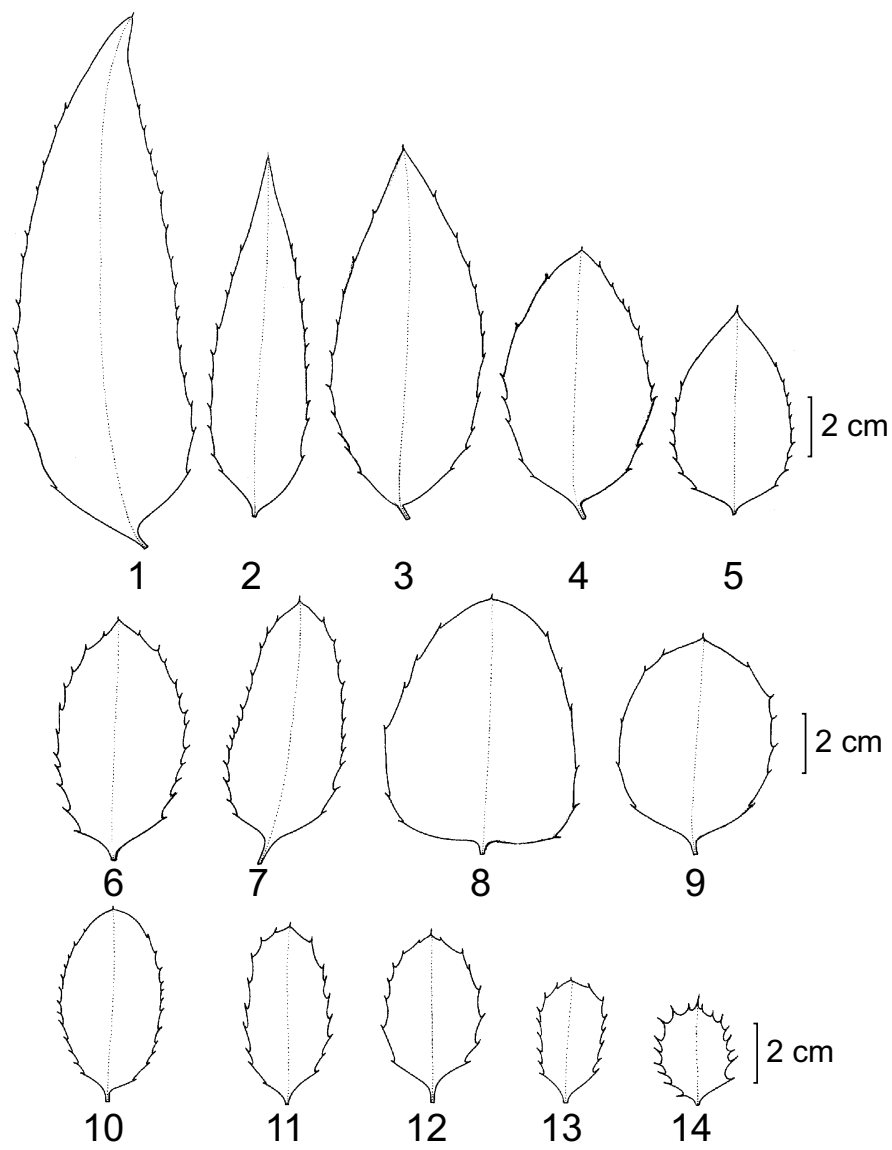

Fig. 9. Variación en los folíolos de Berberis hartwegii Benth. 1 y 2. Cañada de las Avispas, municipio de Jalpan, Querétaro, S. Servín 931 (IEB); 3. 2-3 km al N de La Parada, municipio de Jalpan, Querétaro, S. Servín 93 (IEB); $4.1 \mathrm{~km}$ al NW de El Sabinito, municipio de Landa, Querétaro, H. Rubio 1539 (IEB); 5 y 6. Minas Viejas, municipio de Zimapán, Hidalgo, $S$. Zamudio, R. Ma. Murillo y A. E. Zamudio 13732 (IEB); 7, 10, 11, 12 y 13. Mina San Francisco, municipio de Zimapán, Hidalgo, S. Zamudio, R. Ma. Murillo y A. E. Zamudio 13737 (IEB); 8. Verdosas, municipio de Zimapán, Hidalgo, S. Zamudio, R. Ma. Murillo y A. E. Zamudio 13739 (IEB); 9 y 14. Puerto Ing. Ignacio Isidro Díaz, municipio de Zimapán, Hidalgo, $S$. Zamudio. R. Ma. Murillo y A. E. Zamudio 13734 (IEB). Dibujo de Rogelio Cárdenas. 
de esta especie con frecuencia se encuentran folíolos con el margen fuertemente revoluto. Estas últimas formas fueron descritas como entidades independientes con los nombres de Mahonia paxii Fedde y Mahonia zimapana Fedde. Aquí se considera que todas constituyen un solo taxon muy variable, distribuido ampliamente en la Sierra Madre Oriental.

A continuación se proporciona una descripción completa de la especie tomando en cuenta la variación estudiada y señalando su sinonimia. Debido a que en el herbario de los Reales Jardines Botánicos de Kew existen tres ejemplares de la colecta de Hartweg 272 y ninguno ha sido señalado como holotipo, aquí se elige un lectotipo de entre ellos.

Berberis hartwegii Benth., Pl. Hartweg. 34. 1840. Tipo: At the Contadero between Tula and Sta. Barbara (Tamaulipas), T. Hartweg 272 (lectotipo aquí designado: Kew000407230). Fig. 10.

Mahonia hartwegii (Benth.) Fedde, Bot. Jahrb. 31: 109. 1901.

Odostemon hartwegii (Benth.) Standl., Contr. U.S. Nat. Herb. 23: 270. 1922.

Mahonia paxii Fedde, Bot. Jahrb. 31: 113. 1901. Tipo: Inter Zimapan et la Encarnación (sic en la etiqueta del espécimen dice: Inter Zimapan et al Queretaro?), enero de 1840, C. A. Ehrenberg 1110 (lectotipo aquí designado: HAL-064676, isolectotipos HAL).

Odostemon paxii (Fedde) Standl., Contr. U.S. Nat. Herb. 23: 271. 1922.

Berberis paxii (Fedde) Marroq. \& Laferr., J. Ariz.-Nev. Acad. Sci. 30(1): 54.1997.

Mahonia zimapana Fedde, Bot. Jahrb. Syst. 31: 111. 1901. Tipo: Las Verdosas prope Zimapan, Jan. 1840, C. A. Ehrenberg 1078 (lectotipo aquí designado: B-100244043, isolectotipo HAL-067511).

Berberis zimapana (Fedde) Marroq. \& Laferr., J. Ariz.-Nev. Acad. Sci. 30(1): 54. 1997.

Arbustos o árboles pequeños de 1 a $5 \mathrm{~m}$ de alto; hojas de (10-)15 a $50 \mathrm{~cm}$ de largo, con 7 a 15(-17) folíolos peciolulados, el par inferior más chico que los demás, pecíolo (3-)5 a 15(-45) mm de largo, el limbo lanceolado, ovado, oblongo a ampliamente ovado o ampliamente elíptico, de 2.5 a 12(-19) $\mathrm{cm}$ de largo por (1.5-)2 a $6.5(-10) \mathrm{cm}$ de ancho, ápice agudo, obtuso a redondeado, mucronado espinoso, base cuneada a redondeada, asimétrica, peciólulo de 1 a $5 \mathrm{~mm}$ de largo, margen ondulado, aserrado dentado a entero, con frecuencia fuertemente revoluto, con 5 a 12(-24) espinas por lado, estas de 0.5 a $2.5(-4) \mathrm{mm}$ de largo, venación reticula- 


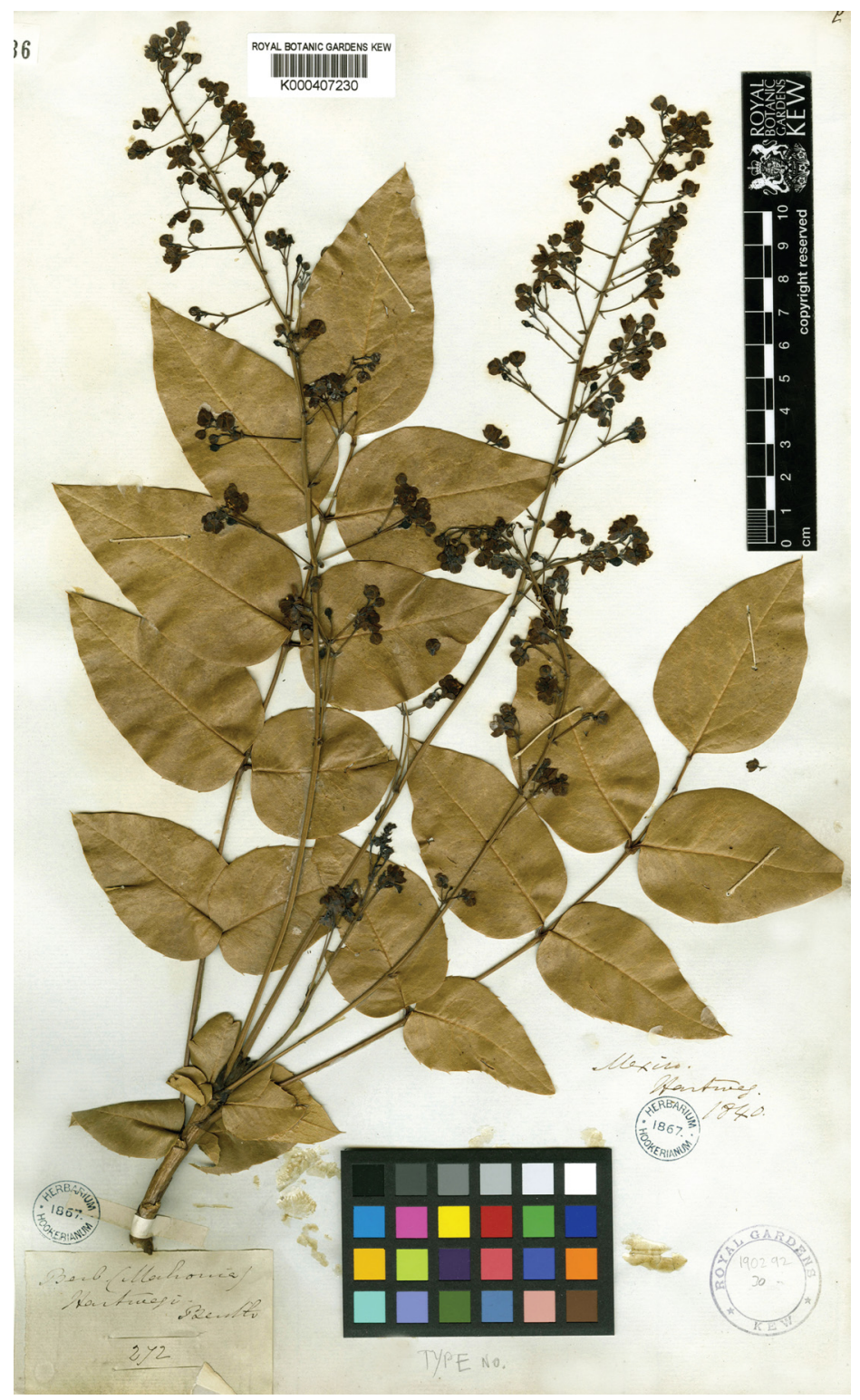

Fig. 10. Lectotipo aquí designado de Berberis hartwegii Benth. Ejemplar de T. Hartweg 272 (K000407230). At the Contadero between Tula and Sta. Barbara. Imagen reproducida con autorización del Herbario de los Reales Jardines Botánicos de Kew. 
da, el nervio medio y los nervios secundarios impresos en el haz, los terciarios y cuaternarios poco conspicuos, todos los nervios resaltados en el envés, coriáceo a subcoriáceo, haz brillante, envés opaco, de color verde oliva a verde-amarillento o café; inflorescencia en panículas abiertas de 15 a 30(-85) cm de largo, brácteas de la inflorescencia triangulares a ovadas, acuminadas o divididas en el ápice, de (5-)8 a $18 \mathrm{~mm}$ de largo por 6 a $12 \mathrm{~mm}$ de ancho, pedúnculo fuerte, de 6 a $18 \mathrm{~cm}$ de largo, pedicelos de (3-)5 a $20 \mathrm{~mm}$ de largo, brácteas florales lanceoladas, de 2 a $13 \mathrm{~mm}$ de largo por 1.5 a $4 \mathrm{~mm}$ de ancho, bractéolas situadas por debajo de la parte media del pedicelo, lanceoladas a ovado-lanceoladas, de 1 a $2 \mathrm{~mm}$ de largo; sépalos externos ampliamente ovados, de 2.5 a $4.5 \mathrm{~mm}$ de largo por 2 a $3 \mathrm{~mm}$ de ancho, sépalos medios obovados, de 5 a $7 \mathrm{~mm}$ de largo por 3 a $5.5 \mathrm{~mm}$ de ancho, sépalos internos obovados, de 5.5 a $8 \mathrm{~mm}$ de largo por 3 a $7 \mathrm{~mm}$ de ancho; pétalos obovados, emarginados, de 4 a 6(-7) $\mathrm{mm}$ de largo por 2.5 a $4 \mathrm{~mm}$ de ancho; estambres de 3 a $5 \mathrm{~mm}$ de largo, sin dientes; fruto globoso, azul pruinoso o glauco, sin estilo, de 8 a $11 \mathrm{~mm}$ de diámetro; semillas (2-)6 a 12, obovoides, de 4.5 a $6 \mathrm{~mm}$ de largo por 2 a $4 \mathrm{~mm}$ de ancho, cafés a café-rojizas.

Arbusto o arbolito escaso en bosques mesófilo de montaña, de pino, de encino, de pino piñonero y en matorral submontano. Alt. 1000-2300 m. Florece de enero a julio y fructifica de abril a octubre.

Especie endémica de la Sierra Madre Oriental, en los estados de Tamps., S.L.P., Qro., Hgo. A pesar de ser una planta que no forma poblaciones densas, no parece enfrentar problemas de supervivencia.

Nombres comunes: cigarrillo, chochoco, laurel cimarrón, palo amarillo, xoxoco.

Ejemplares revisados: Hidalgo: Eloxochitlán, $4.5 \mathrm{~km}$ al E, municipio de Eloxochitlán, O. Alcántara y R. Mayorga 2963 (MEXU); $10 \mathrm{~km}$ al N de Jacala sobre la Carretera Federal 85, México - Nuevo Laredo, municipio de Jacala, 23.II.1991, C. P. Rodríguez y Z. C. Ugalde s.n. (MEXU); carretera México - Nuevo Laredo, km 190, a $9 \mathrm{~km}$ de Jacala, municipio de Jacala, A. Díaz-Vilchis 7 (MEXU); carretera 85 México - Nuevo Laredo, km 190, municipio de Jacala, M. P. Casillas-Trejo 2 (MEXU); cerro al W de Agua Fría Grande, cerca de Jacala, municipio de Jacala, $L$. González-Quintero 910 (ENCB); Highway 85, km 87 north of juntion with Highway 45, municipio de Zimapán, A. C. Gibson \& L. C. Gibson 2651 (ENCB, MEXU); Minas Viejas, near km 255 on Highway between Zimapan and Jacala, municipio 
de Zimapán, H. E. Moore Jr. \& C. E. Wood Jr. 3853 (MEXU); Minas Viejas, 47 $\mathrm{km}$ al NE de Zimapán, municipio de Zimapán, R. Hernández M. y D. Rodríguez B. $5082 a$ (ENCB, MEXU, XAL); Minas Viejas, municipio de Zimapán, S. Zamudio, R. Ma. Murillo y A. E. Zamudio 13732 (IEB); Minas Viejas, municipio de Zimapán, 14.I.1978, E. Guízar-Nolazco et al. s.n. (IBUG); $40 \mathrm{~km}$ al N de Zimapán, carretera a Jacala, municipio de Zimapán, R. Torres 14456 (MEXU); al NE de Zimapán, km 248 de la carretera México-Laredo, municipio de Zimapán, H. Puig 4414 (ENCB); $20 \mathrm{~km}$ al NE de Zimapán sobre la carretera a Jacala, municipio de Zimapán, $J$. Rzedowski 23074 (ENCB); norte de Zimapán, municipio de Zimapán, M. F. Robert 439 (ENCB); cañón del Arroyo Tolimán, municipio de Zimapán, S. Zamudio y E. Pérez 9122 (IEB); Verdosas, municipio de Zimapán, S. Zamudio, R. Ma. Murillo y A. E. Zamudio 13739 (IEB); mina San Francisco, $\pm 14.3 \mathrm{~km}$ por la brecha a Verdosas, municipio de Zimapán, S. Zamudio, R. Ma. Murillo y A. E. Zamudio 13737 (IEB); Puerto Ingeniero Isidro Díaz, municipio de Zimapán, S. Zamudio, R. Ma. Murillo y A. E. Zamudio 13734 (IEB). Sin municipio definido: between Hilo Juanico and Barranca Seca on trail from Jacala to Pacula, H. E. Moore Jr. y C. E. Wood Jr. 3832 (MEXU).

Querétaro: alrededores de El Saucito, al N de Valle Verde, municipio de Jalpan, E. Carranza e Y. Ramírez 7335, 7336 (IEB); 2-3 km al N de Valle Verde (La Parada), municipio de Jalpan, S. Servín 93 (IEB); $3 \mathrm{~km}$ al ENE de Valle Verde, municipio de Jalpan, E. Carranza y A. Blanco 6061 (IEB); 1-2 km al E de Valle Verde (La Parada), municipio de Jalpan, S. Servín 2 (IEB); alrededores de Valle Verde (La Parada), municipio de Jalpan, E. Carranza 2353 (IEB); Cañada de las Avispas, 5-6 km al S de San Juan de los Durán, municipio de Jalpan, S. Servín 931 (IEB); extremo $\mathrm{N}$ del Llano Chiquito, municipio de Landa, S. Zamudio, G. Ocampo y A. Reznicek 11471 (IEB); Joya del Cerro Prieto, entre la Lagunita de San Diego y el Llano Chiquito, municipio de Landa, S. Zamudio y E. Pérez 10189 (IEB); $\pm 3.5 \mathrm{~km}$ al ENE de la Lagunita de San Diego, municipio de Landa, E. Carranza, E. Pérez y J. Á. Villarreal 5389 (IEB); la Mesa, $\pm 2 \mathrm{~km}$ al SE de la Lagunita de San Diego, municipio de Landa, S. Zamudio y V. Steinmann 12386 (IEB); la Lagunita de San Diego, municipio de Landa, S. Zamudio y V. Steinmann 12374 (IEB); $10 \mathrm{~km}$ al SW de El Madroño, sobre el camino a Landa, municipio de Landa, J. Rzedowski 46775 (IEB); $8 \mathrm{~km}$ al NE de la Lagunita por la carretera a Xilitla, municipio de Landa, $S$. Zamudio 6281 (IEB); Rincón de La Trampa, $\pm 2 \mathrm{~km}$ al W de Acatitlán de Zaragoza, municipio de Landa, E. González 52 (IEB); Puerto del Gato, $\pm 3 \mathrm{~km}$ al W de Acatitlán de Zaragoza, municipio de Landa, E. González 384 (IEB); aproximadamente $6 \mathrm{~km}$ al S de La Lagunita rumbo a Tilaco, municipio de Landa, E. Carranza 609 
(IEB); 8.5-9 km por la carretera la Lagunita - Tilaco, municipio de Landa, S. Zamudio y A. Ibarra 12718 (IEB); $\pm 9 \mathrm{~km}$ de la Lagunita camino a Tilaco, municipio de Landa, E. Carranza e I. Silva 6361 (IEB); km 10 de la brecha entre la Lagunita y Tilaco, municipio de Landa, S. Zamudio 6332 (IEB); $10 \mathrm{~km}$ de la Lagunita, por el camino hacia Tilaco, municipio de Landa, A. Herrera 44 (IEB); aproximadamente 4-5 km al NW de Tilaco, municipio de Landa, E. Carranza 1597 (IEB); 1 km al NW de El Sabinito, municipio de Landa, H. Rubio 1539 (IEB); $1.5 \mathrm{~km}$ al SE de el Sabinito, municipio de Landa, H. Rubio 429 (IEB); $11 \mathrm{~km}$ al SE de Agua Zarca, por el camino a Pisaflores, municipio de Landa, S. Zamudio 6261 (IEB); 1 km al NW de el Rincón, municipio de Landa, H. Rubio 2233 (IEB); brecha hacia La Redonda, municipio de San Joaquín, R. Hernández M., J. Orozco y C. Orozco 10495 (QMEX).

San Luis Potosí: Tamasopo, municipio de Tamasopo, C. G. Pringle 2566 (MEXU); cerro de La Silleta, cerca de Xilitla, municipio de Xilitla, L. Paray 693 (ENCB); Cerro Prieto, west Xilitla, municipio de Xilitla, A. J. Sharp 46285 (MEXU); $\pm 5 \mathrm{~km}$ al NW de Tlamaya, municipio de Xilitla, J. Rzedowski 7205 (ENCB, IBUG); $5 \mathrm{~km}$ al $\mathrm{N}$ de Soledad de Zaragoza, municipio de Xilitla, J. Rzedowski 7108 (ENCB).

Tamaulipas: camino a Ojo de Agua del Indio, $7 \mathrm{~km}$ al $\mathrm{W}$ de Rancho del Cielo y $18 \mathrm{~km}$ al E de Gómez Farías, municipio de Gómez Farías, F. González-Medrano et al. 4271 (MEXU); El Julilo, $18 \mathrm{~km}$ al W de El Encino, municipio de Gómez Farías, F. González-Medrano, P. Hiriart y P. Hiriart 13151 (MEXU); Rancho del Cielo, arriba de Gómez Farías, municipio de Gómez Farías, A. Gómez-Pompa 2033 (MEXU); entre Alta Cima y San José, municipio de Gómez Farías, F. GonzálezMedrano et al. 3343 (MEXU); Casa de Piedra, $3 \mathrm{~km}$ al NW de la estación biológica Canindos, 4 km al NW de San José, municipio de Gómez Farías, L. Hernández 2988 (MEXU); NW of Gómez Farías in the Sierra de Guatemala, area of Rancho del Cielo, L. Woodruff, et al. 173 (MEXU, XAL); ejido San José, municipio de Gómez Farías, R. Noriega T. 7 (MEXU); Rancho del Cielo, municipio de Gómez Farías, $H$. Puig 3272 (ENCB); camino a Rancho del Cielo, municipio de Gómez Farías, H. Puig 5166, 5181 (ENCB); entre Gómez Farías y Rancho del Cielo, municipio de Gómez Farías, F. Vázquez B. 828 (XAL); Rancho del Cielo, municipio de Gómez Farías, $S$. Avendaño y $H$. Narave 1652 (XAL).

En esta especie se incluyen los ejemplares descritos por Fedde (1901) como Berberis paxii y B. zimapana, ya que no se encontraron rasgos distintivos de suficiente peso que justifiquen su reconocimiento como entidades independientes. 
En casi todos los caracteres se encuentra una amplia sobreposición y las características más distintivas están relacionadas con la forma de los folíolos, que en $B$. hartwegii se describen como subcoriáceos, ovado-lanceolados con el ápice agudo mucronado, mientras que en B. paxii y B. zimapana, se refieren como coriáceos, ovados con el ápice redondeado a muy ampliamente cuneado-mucronado. La observación de los ejemplares tipo existentes y de nuevos materiales en las localidades de las colectas originales o en sus cercanías ha permitido confirmar que las formas involucradas en estas dos especies son parte de la amplia variación observada en Berberis hartwegii a través de su área de distribución, como se puede apreciar en la Fig. 9.

Por otro lado, como ya se ha indicado Berberis hartwegii es muy parecido a $B$. pallida, por lo que con frecuencia el material de las dos especies se encuentra confundido y mezclado en los herbarios mexicanos. Una forma segura de diferenciarlos es comparando la venación de los folíolos en el haz; en ambos taxa la venación es reticulada, pero en $B$. hartwegii los nervios están impresos o hundidos en la superficie de la lámina, por lo que son poco conspicuos; en cambio, en Ber-

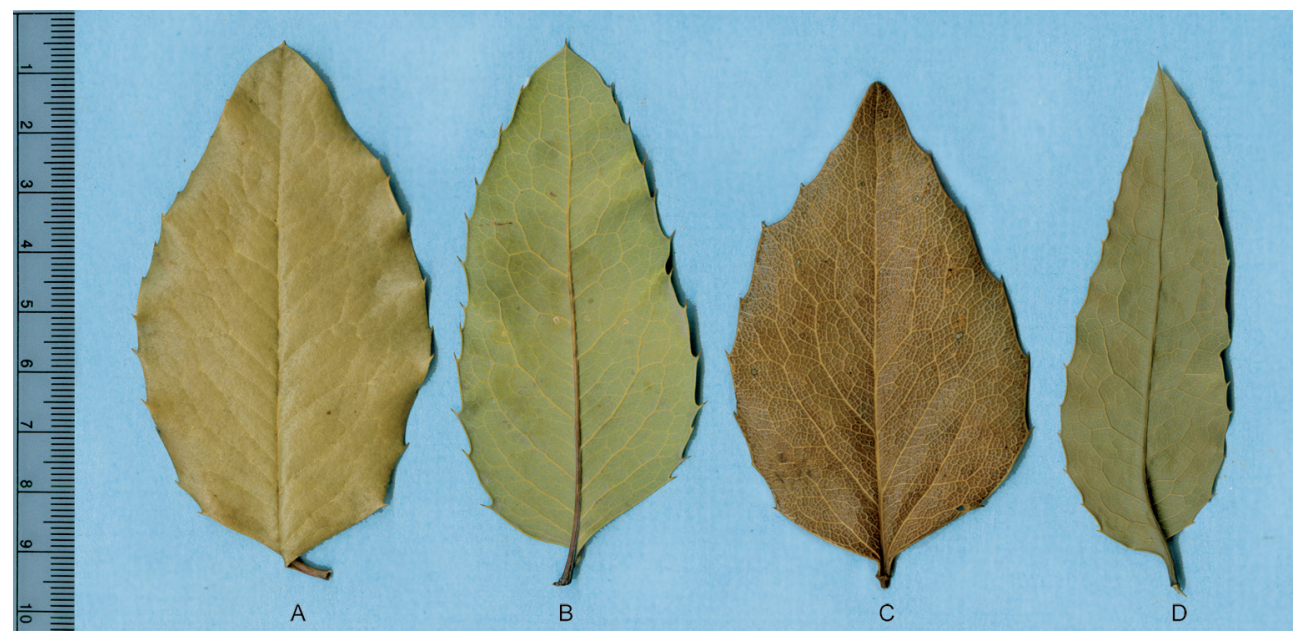

Fig. 11. Comparación de patrón de venación de los folíolos entre Berberis hartwegii Benth. y B. pallida Hartweg ex Benth. A y B. Berberis hartwegii, con venas impresas o hundidas en la superficie de la lámina del folíolo en el haz y venas resaltadas en el envés; C y D. Berberis pallida con venas fuertemente reticuladas y resaltadas en ambas caras, pero más notorias en el haz que en el envés. 
beris pallida se distinguen notoriamente resaltados (Fig. 11). Adicionalmente en $B$. hartwegii se aprecia un mayor número de dientes por lado y es común observar los folíolos enrollados hacia atrás, ya que el margen es fuertemente revoluto. Otra diferencia entre estas dos especies es que en B. pallida los estambres son dentados, mientras que en $B$. hartwegii tales dientes están ausentes. Ya que ambas especies ocupan habitats muy parecidos en la Sierra Madre Oriental, es muy probable que cuando coinciden en su distribución, se formen híbridos entre ellas, los que son difíciles de separar. Así por ejemplo, el espécimen de Minas Viejas, municipio de Zimapán, colectado por S. Zamudio et al. 13732, presenta características intermedias entre los dos taxa.

\section{AGRADECIMIENTOS}

Deseo dar gracias por la ayuda de Armando Butanda, Gilberto Ocampo y Lourdes Rico en la consecución de literatura en revistas especializadas que no son de fácil acceso en México. Al Dr. Jerzy Rzedowski agradezco su ayuda en la traducción de textos del alemán al español, la revisión crítica del manuscrito y sus atinadas sugerencias; a Rogelio Cárdenas por la elaboración de los dibujos; a los encargados de los Herbarios B, ENCB, HAL, IBUG, IEB, MEXU, K, QMEX y XAL por las facilidades otorgadas para consultar sus colecciones. Las imágenes de los ejemplares tipo depositados en B, HAL y K son publicadas con autorización de los respectivos herbarios.

\section{LITERATURA CITADA}

Ahrendt, L. W. A. 1961. Berberis and Mahonia. A taxonomic revision. Jour. Linn. Soc. Bot. 57: 1-410.

Bentham, G. 1839-1857. Plantae Hartwegianae. Londres. 393 pp.

Fedde, F. 1901. Versuch einer Monographie der Gattung Mahonia. Bot. Jahrb. 31: 30-133.

Hemsley, W. B. 1879-1888. Botany. In: Godwin, F. D. y O. Salvin (eds.). Biologia CentraliAmericana. Vol. 1. R. H. Porter. Londres. pp. 22-24.

Marroquín, J. S. 1972. A monographic study of the genus Berberis L. in Mexico. Thesis of doctor in philosophy degree. Graduate School of Arts and Sciences. Northeastern University. Boston, Massachusetts. 177 pp.

Marroquín, J. S. 1993. Berberidaceae. Flora de Veracruz 75: 1-16.

McVaugh, R. 1970. Introduction to the facsimile reprint of George Bentham's "Plantae Hartwegianae". J. Cramer. Lehre. 102 pp. 
Schiede, C. J. W. 1829. Zweiter Bericht über die Gegend um Jalapa und Excursion auf den Volcan de Orizaba. Linnaea 4(2): 212-236.

Schlechtendal, D. F. L. y A. de Chamisso. 1830. Plantarum Mexicanarum. Linnaea 5: 206236.

Schlechtendal, D. F. L. 1835-1836. De plantis Mexicanis. Linnaea 10: 233-254.

Schlechtendal, D. F. L. 1854. Über eine neue Mahonia nebst Bemerkungen über einige ältere. Bot. Zeit. 12: 651-656.

Schultes, J. A. y J. H. Schultes. 1830. Systema vegetabilium. Vol. 7. Stuttgardtiae. Sumtibus J. G. Cottae. p. 1616.

Stafleu, F. A. y R. S. Cowan. 1985. Taxonomic literature. A selective guide to botanical publications and collections with dates, commentaries and types. Regnun vegetabilie. 2a. ed. Vol. 5. Bohn, Scheltema \& Holkema. Utrecht. 1066 pp.

Standley, P. C. 1922. Trees and shrubs of Mexico. Contr. U.S. Nat. Herb. 23(2): 268-273.

Taylor, N. P. 2004. Mahonia pallida, Berberidaceae. Curtis’s Bot. Mag. 21(3): 182-184. 